\title{
Statistical analysis of the Bacterial Carbohydrate Structure Data Base (BCSDB): Characteristics and diversity of bacterial carbohydrates in comparison with mammalian glycans Stephan Herget ${ }^{* \dagger 1}$, Philip V Toukach ${ }^{\dagger 2}$, René Ranzinger ${ }^{1}$, William E Hull${ }^{1}$, Yuriy A Knirel ${ }^{2}$ and Claus-Wilhelm von der Lieth ${ }^{1}$
}

\begin{abstract}
Address: ${ }^{1}$ Core Facility: Molecular Structure Analysis (W160), German Cancer Research Center, Heidelberg, Germany and ${ }^{2}$ N.D. Zelinsky Institute of Organic Chemistry, Russian Academy of Sciences, Moscow, Russia

Email: Stephan Herget* - s.herget@dkfz.de; Philip V Toukach - never@vilvit.net; René Ranzinger - r.ranzinger@dkfz.de; William E Hull -w.hull@dkfz.de; Yuriy A Knirel - knirel@ioc.ac.ru; Claus-Wilhelm von der Lieth - m.frank@dkfz.de

* Corresponding author †Equal contributors
\end{abstract}

Published: II August 2008

BMC Structural Biology 2008, 8:35 doi:1 0.1 186/1472-6807-8-35

This article is available from: http://www.biomedcentral.com//472-6807/8/35

(c) 2008 Herget et al; licensee BioMed Central Ltd.

This is an Open Access article distributed under the terms of the Creative Commons Attribution License (http://creativecommons.org/licenses/by/2.0), which permits unrestricted use, distribution, and reproduction in any medium, provided the original work is properly cited.
Received: 26 February 2008

Accepted: II August 2008

\begin{abstract}
Background: There are considerable differences between bacterial and mammalian glycans. In contrast to most eukaryotic carbohydrates, bacterial glycans are often composed of repeating units with diverse functions ranging from structural reinforcement to adhesion, colonization and camouflage. Since bacterial glycans are typically displayed at the cell surface, they can interact with the environment and, therefore, have significant biomedical importance.

Results: The sequence characteristics of glycans (monosaccharide composition, modifications, and linkage patterns) for the higher bacterial taxonomic classes have been examined and compared with the data for mammals, with both similarities and unique features becoming evident. Compared to mammalian glycans, the bacterial glycans deposited in the current databases have a more than tenfold greater diversity at the monosaccharide level, and the disaccharide pattern space is approximately nine times larger. Specific bacterial subclasses exhibit characteristic glycans which can be distinguished on the basis of distinctive structural features or sequence properties.

Conclusion: For the first time a systematic database analysis of the bacterial glycome has been performed. This study summarizes the current knowledge of bacterial glycan architecture and diversity and reveals putative targets for the rational design and development of therapeutic intervention strategies by comparing bacterial and mammalian glycans.
\end{abstract}

\section{Background}

Natural glycans are known to take part in many key biological processes such as cell adhesion, recognition, receptor activation or signal transduction, and they also exhibit major structural functions in both bacteria and plants. In addition, bacterial glycans act as virulence, osmoprotec- tion and desiccation protection factors [1]. The diversity of structures within the mammalian glycome seems limited and is well described in the literature [2]. On the other hand, the bacterial glycome exhibits greater diversity, stemming largely from the distinct cell wall architecture of these organisms. 
The cell envelope of either Gram-positive or Gram-negative bacteria is based on peptidoglycan, a polymer in which polysaccharide chains are cross-linked with short peptide chains [3]. Gram-negative bacteria possess an additional outer membrane that is composed of a lipopolysaccharide-protein complex. Gram-positive bacteria have no outer membrane, but the peptidoglycan wall is thicker (> $30 \mathrm{~nm}$ vs. $10 \mathrm{~nm}$ in Gram-negative bacteria) and contains polysaccharides with teichoic acids attached (a carbohydrate polymer containing alditols and phosphodiester linkages).

Both Gram-positive and Gram-negative bacteria produce extracellular polysaccharides, present either as a discrete capsule covalently attached to the cell envelope or as a slime weakly bound to the cell surface. These various glycoconjugates and polysaccharides on the surface of the cell often contain the antigenic determinants that initiate an immunogenic response in a host. In addition, these surface carbohydrates provide recognition elements for pathogens such as bacteriophages.

The lipopolysaccharide of Gram-negative bacteria contains lipid A, a phosphorylated GlcN-GlcN disaccharide moiety, $\mathrm{N}$ - and $\mathrm{O}$-acylated with fatty acids which anchor the molecule in the outer leaflet of the outer membrane. Lipid A is covalently linked to a heteropolysaccharide which interacts with the environment and consists of an inner core (commonly containing Kdo (3-deoxy-Dmanno-oct-2-ulosonic acid) and manno-heptoses) and an outer $O$-specific chain, a complex polysaccharide which determines the serological or antigenic properties of the lipopolysaccharide $[4,5]$. These so-called $O$-antigens are mainly heteropolymers containing a large variety of residues (mainly monosaccharides, but also alditols, amino acids, etc.). These components, together with the capsular polysaccharides (K-antigens [6,7]), can elicit an immune response in higher organisms.

The structures of the various carbohydrate antigens are unique, often being characterized by repeating units in the polymer structure. Indeed, all types of monosaccharides, including L-rhamnose (6-deoxy-L-mannose) and L-fucose (6-deoxy-L-galactose), are found in bacteria, together with rarer, modified sugars, such as 3,6-dideoxyhexoses and Kdo. Knowledge of the structures of surface carbohydrates and their variations is required for understanding how cellular recognition, adhesion, and the immune response operate at the molecular level. This understanding provides a basis for the design of synthetic carbohydratebased vaccines, diagnostic agents, and immunostimulators. Certain fragments of bacterial polysaccharides, in the form of appropriate glycoconjugates, are known to act as vaccines [8].
Carbohydrates represent the most diverse class of biopolymers, and there is growing interest in the study and analysis of this diversity and its biomedical significance. For example, vertebrate glycan variability is assumed to act as a barrier that prevents the spread of an infection within a given population [9]. Although it is widely known that the diversity of carbohydrates is much greater in bacteria than in mammals, no systematic attempt has been undertaken to examine the diversity of bacterial carbohydrates in detail. The structures deposited in glycoscience databases have been only sporadically evaluated. However, statistical structure-oriented investigations using carbohydrate databases were proven to be useful for immunochemical research and serotyping [10]. Systematic analysis of all publicly available data will not only expand our general knowledge and understanding of the complexity of glycans in biological systems but will also offer a framework for the design of more comprehensive high-throughput screening methods or devices.

Comprehensive data concerning carbohydrate diversity within the entire bacterial world will be useful for the classification of bacteria according to their glycan structures and facilitate the search for the most widespread carbohydrate markers of various bacterial taxonomic groups. These markers are critical for medical applications, and a simple ranking by abundance is a good starting point for the design of synthetic biologically-active carbohydrates and for corresponding immunological studies. In particular, the statistics of monomer composition reveal potential taxonomic markers and also simplify the creation of carbohydrate microarrays by providing candidates for spotting [11].

A one-enzyme-class/one-saccharide-linkage paradigm applies for almost all individual steps of glycan biosynthesis. Accordingly, complete information on the diversity of disaccharide fragments allows one to describe the diversity of the glycosyltransferases expressed in individual taxonomic groups, and these enzymes may become potential targets for antimicrobial treatment.

For this study we performed statistical analyses of the Bacterial Carbohydrate Structure Data Bank (BCSDB), the largest database for bacterial glycans containing nearly all known bacterial glycan structures published up to 2007 [12]. For comparison the mammalian glycans documented in the GLYCOSCIENCES.de database [13] (derived mainly from CarbBank [14]) have also been examined. The properties analyzed include glycan size, branching, and charge density, as well as the frequency of occurrence of specific monosaccharide residues, residue pairs and their linkage configurations. Precise definitions for the terminology used in this study can be found in the Methods section. 


\section{Results and Discussion Distribution of carbohydrate structures among taxonomic groups}

We first examined the number of sequences found in the BCSDB and GLYCOSCIENCES.de for various taxonomic ranks (class, order, family). Where possible, the taxonomic relationships were traced using the NCBI taxonomy database [15]. The GLYCOSCIENCES.de database currently contains a total of 23120 glycan and glycoconjugate records, of which 13704 records for diverse animal, plant, bacteria and fungi classes have some information concerning taxonomy. In the BCSDB there are a total of 8504 records for bacteria only, and 8479 of these contain information concerning taxonomy. These numbers may include multiple records for a given glycan when the same glycan is reported for more than one species. Note that not all taxonomic classes are represented in the databases and that for bacterial glycans there is considerable overlap between the two databases.

If we now consider the two databases combined, there are a total of 13775 nonredundant carbohydrate records which include taxonomic information. The distribution of these records among various taxonomic classes is shown both numerically and schematically in Fig. 1. A more detailed breakdown of the distribution can be found in the additional material [see Additional file 1].
The taxonomic class Mammalia is found to have 4739 assigned sequence/taxon pairs, of which 2118 are of human origin (family Hominidae). All other animal or plant classes in the database have less than 350 pairs. The category "unresolved" refers to the 1482 records for which the source is defined but the specific taxonomic class could not be traced automatically using the NCBI. Only about half of the bacterial phyla are represented in the BCSDB with a total of 6098 sequence/taxon pairs, and nine classes have less than 10 records. Note that the number of carbohydrates or glycoconjugates registered for a given taxonomic class does not necessarily reflect its species diversity, but more likely the intensity with which the class has been studied. Thus, the apparent diversity of carbohydrates in the various taxonomic classes reflects to a large part the information bias in the published literature, and this situation must be kept in mind when making conclusions based on the distributions of properties discussed below.

In the combined databases there are a total of 12659 records in the category "no taxonomy" which means that either no information concerning the taxonomy of the source is available or that the carbohydrate is not of purely natural origin. These records were not included in Fig. 1 and were not used in the following analyses.

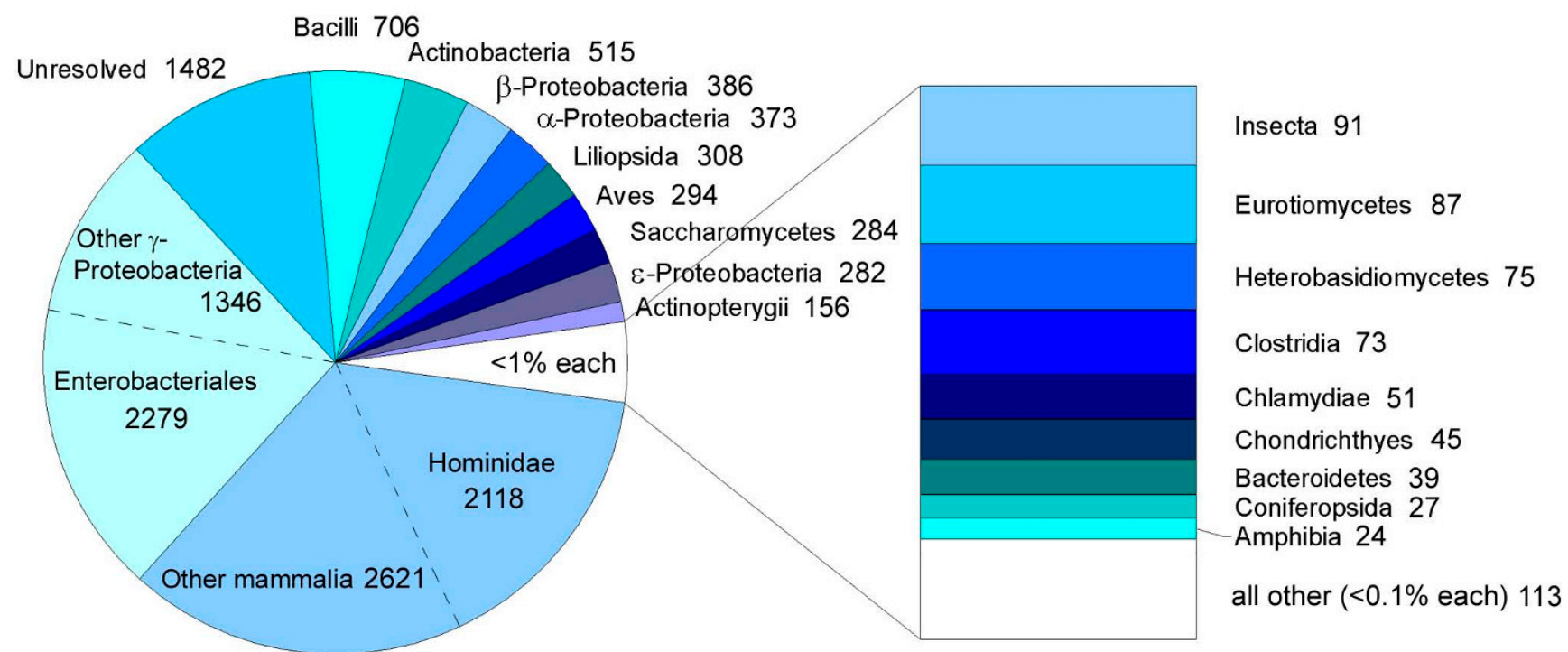

Figure I

Distribution of carbohydrate sequences for various taxonomic classes. For the combined BCSDB and GLYCOSCIENCES.de databases the pie chart sector areas correspond to the distribution (in percent) of the I 3775 assigned sequences within the taxonomic classes shown, while the labels give the absolute numbers of sequences. The white pie sector contains all classes which each have $<1 \%$ of the total assigned records. This category is expanded in the bar chart at the right, where the bottom block "all other" contains all classes which each have $<0.1 \%$ of the assigned records. Class names ending with ...opsida or ...mycetes correspond to plants or fungi, respectively; Actinopterygii contains fish while Chondrichthyes contains sharks. 
Choice of taxonomic datasets for statistical comparisons For the following more detailed statistical comparisons, we defined two sets of taxonomic groups, considering both biological and coverage aspects. Taxonomy Set 1 (Table 1) was defined to provide an overview of the total content of the two databases used for the general comparison of bacterial and mammalian carbohydrates, taking into account the fact that bacterial carbohydrates frequently contain repeating units while mammalian sequences usually do not. Thus, Set 1 contains three taxonomic groups: all mammalian carbohydrates, all bacterial carbohydrates with nonrepeating sequences (oligomers), and all bacterial sequences with repeating units (polymers).

For comparisons within the taxonomic domain Bacteria, we defined a more detailed taxonomy Set 2 (Table 2), which includes two classes of Gram-positive bacteria (Actinobacteria and Bacilli) and the various classes of the phylum Proteobacteria. The largest of these classes, the $\gamma$ Proteobacteria, has been further subdivided in Set 2 into the major order Enterobacteriales and a subset containing all other $\gamma$-Proteobacteria. The class $\delta$-Proteobacteria (with only 2 records) has been combined with the $\varepsilon$-Proteobacteria.

In order to obtain meaningful statistics, only those taxonomic groups are compared for which at least 200 carbohydrate sequences are available. For this reason the classes Chlamydiae, Clostridia, and Bacteroidetes, for example, have not been included in Set 2. Note that in Tables 1 and 2 the total number of unique carbohydrate sequences in each group is listed, and these sets were utilized in all subsequent analyses.

\section{Carbohydrate size, branching and charge density}

Frequency distributions for general measures of molecular size, topology (branching) and mean charge density have been calculated for the carbohydrate sequences comprising the various taxonomic groupings described by Set 1 and Set 2 (Tables 1 and 2). In each case the distributions are normalized to the total number of sequences in each taxonomic group and expressed as percentages within each group. In Fig. 2A distributions for the number of monosaccharides per sequence unit (either the entire carbohy- drate sequence for oligomers or the repeating unit for polymers, see Definition 4 in the methods) are shown for bacteria vs. mammals (taxonomy Set 1 ). The distribution is relatively broad for mammals with mean and median values, respectively, of 8.17 and 8 monomers per sequence, while for bacteria the distribution shows a narrow peak at 4-5 monomers for both oligomers (mean: 5.94 median: 5) and for the repeating unit of polymers (mean: 4.17, median: 4). However, oligomers show a significant population of sequences with 8-15 monomers while the distribution for polymers essentially ends at 9 monomers per unit. Of course, the total length of a polymeric sequence with multiple units may very well exceed the maximum length of oligomers. Naturally occurring oligomers may also be longer than the sequences reported in the databases since the process of extracting and isolating a glycan may result in partial digestion and loss of residues.

In Fig. 2B the distributions of the size parameter for the bacterial groups defined in taxonomy Set 2 are found to differ considerably. Narrow distributions with essentially a single prominant peak are found for Actinobacteria (mean: 4.51, median: 3), Bacilli (mean: 5.18, median: 5) and the order Enterobacteria (mean: 5.18, median: 6) with peaks at ca. 2.5, 5.5 and 4.5 residues, respectively. The various other classes of Proteobacteria have broader distributions with more or less pronounced multiple peaks, e.g. at 3,8 and 11 residues for the $\delta, \varepsilon$-Proteobacteria group.

The number of branching points per carbohydrate residue can be considered to be a branching index which reflects the complexity of carbohydrate topology. Fig. 3A demonstrates that $22 \%$ of all mammalian and $50 \%$ of all bacterial sequences are linear (branching index $=0$ ). However, for the individual bacterial groups of taxonomy Set 2, the percentage of linear structures ranges from $30 \%$ to $78 \%$ (Fig. 3B). A general feature of all branching point graphs in Fig. 3 is a peak in the distribution at a branching index of 0.2 - 0.3, which corresponds to carbohydrate sequences with one branching point for every three to five monosaccharide residues. This peak in the distribution is weak for Actinobacteria and $\alpha$-Proteobacteria but strong for mammals, Bacilli, and other Proteobacteria.

Table I: Definition of taxonomy Set I.

\begin{tabular}{lcl}
\hline Group Name & Number of sequences* & Explanation \\
\hline Mammalia (class) & 3328 & $\begin{array}{l}\text { Total number of different carbohydrate (glycan) sequences for Mammalia, as registered in } \\
\text { GLYCOSCIENCES.de. } \\
\text { Total number of different repeating units in the polymeric carbohydrate (glycan) sequences for } \\
\text { all bacteria registered in the BCSDB. } \\
\text { Total number of different oligosaccharide sequences (nonrepeating units) for all bacteria } \\
\text { Bacteria (oligomers) }\end{array}$ \\
$\begin{array}{ll}\text { registered in the BCSDB. }\end{array}$
\end{tabular}

* For each group each unique carbohydrate sequence was counted only once; a given sequence may occur in more than one taxonomic group. 
Table 2: Definition of taxonomy Set 2.

\begin{tabular}{lcc}
\hline Bacterial Class* & Number of sequences** & Gram reaction \\
\hline Actinobacteria & 395 & + \\
Bacilli & 640 & + \\
$\alpha$-Proteobacteria & 324 & - \\
$\beta$-Proteobacteria & 365 & - \\
$(\gamma$-Proteobacteria) & $(3305)$ & - \\
Enterobacteriales [order] & 2087 & 1218 \\
other $\gamma$-Proteobacteria [orders] & 284 & $(2)$ \\
$\delta / \varepsilon-$ Proteobacteria & $(282)$ & - \\
$(\delta$-Proteobacteria) & $(\varepsilon$-Proteobacteria) & \\
\hline
\end{tabular}

* The groups used to define Set 2 are those with more than 200 sequences and are listed without parentheses.

** For each class each unique carbohydrate sequence was counted only once; a given sequence may occur in more than one taxonomic group.

Finally, the mean charge density parameter (max. electric charge possible for all ionizable groups divided by the number of carbohydrate residues in a sequence unit) is shown in Fig. 4 for taxonomy Set 1 and Set 2. About 58\% of mammalian sequences and $47 \%$ of all bacterial carbohydrate sequence units have no net charge (Fig. 4A). For the bacterial groups of Set 2, the frequency of sequence units with no net charge ranges from ca. $32 \%$ for $\beta$-Proteobacteria to $77 \%$ for Actinobacteria (Fig. 4B). All other sequences have a net negative charge due to carboxyl, phosphate or sulfate groups, for example. The distribu- tions for mammals and Bacilli both have peaks at charge densities of -0.2 and -0.5 ; the $\delta, \varepsilon$-Proteobacteria distribution exhibits a single broad peak at ca. -0.3 while the other bacterial distributions have multiple peaks at $-0.3,-0.5$, 0.7 and -1.0 (Fig. 4B).

\section{Monosaccharide diversity}

For mammals and even more so for bacteria, the diversity of the monosaccharides used as the building blocks of carbohydrate sequences is significantly larger than that for the residues in proteins or nucleic acids. From the GLY-
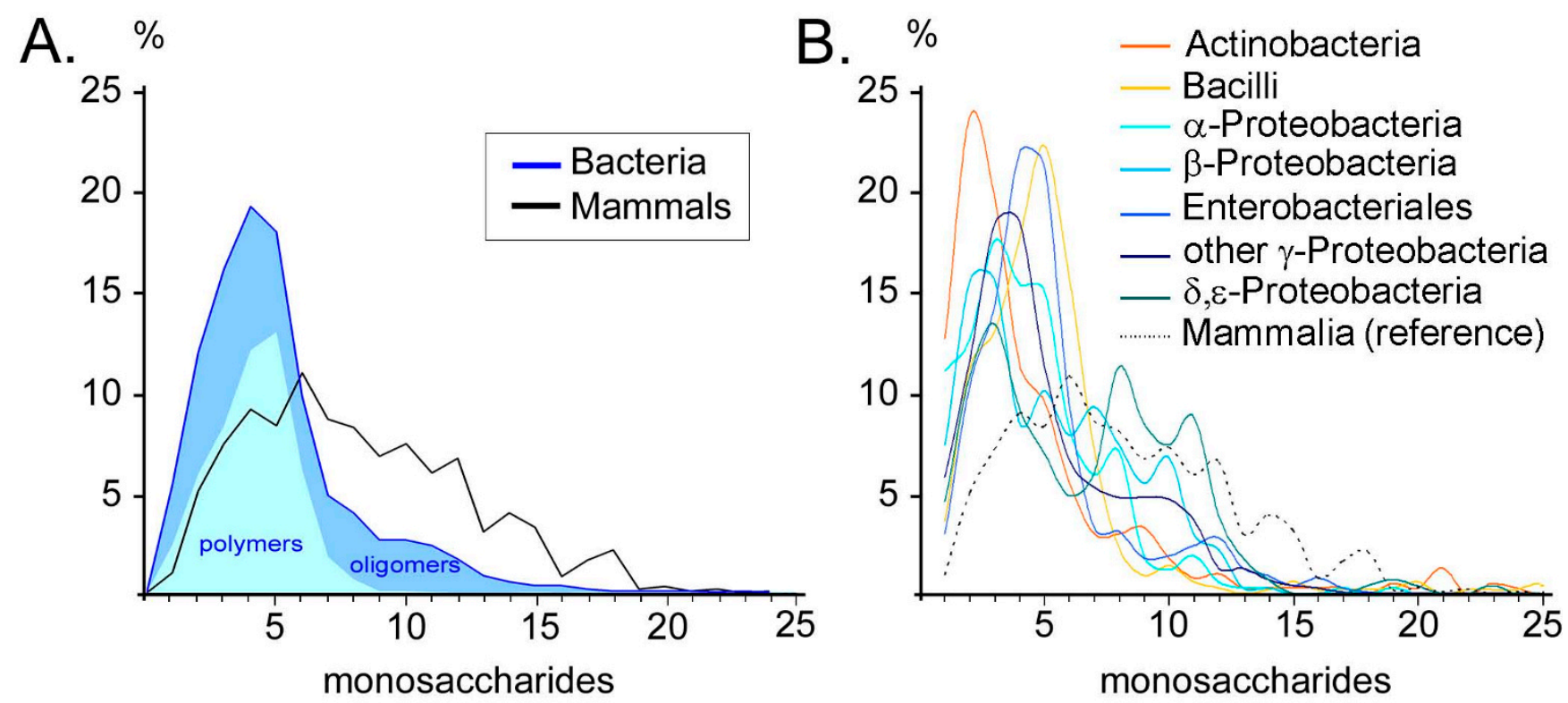

Figure 2

Size distribution of carbohydrate sequence units. The normalized frequency distribution for total carbohydrate residue count per sequence unit is shown in percent of total sequences for each taxonomic group. A. For taxonomy Set I the solid blue curve represents the cumulative values for bacterial oligomers (blue-shaded region) and the repeating units of polymers (cyan region) in comparison with mammals (black curve). B. The frequency distribution for carbohydrate residue count per sequence unit is shown for each of the bacteria groups defined in taxonomy Set 2, using the color coding defined in the legend. For comparison the dotted curve shows the distribution for mammals. The curves are smoothed for visual clarity. 

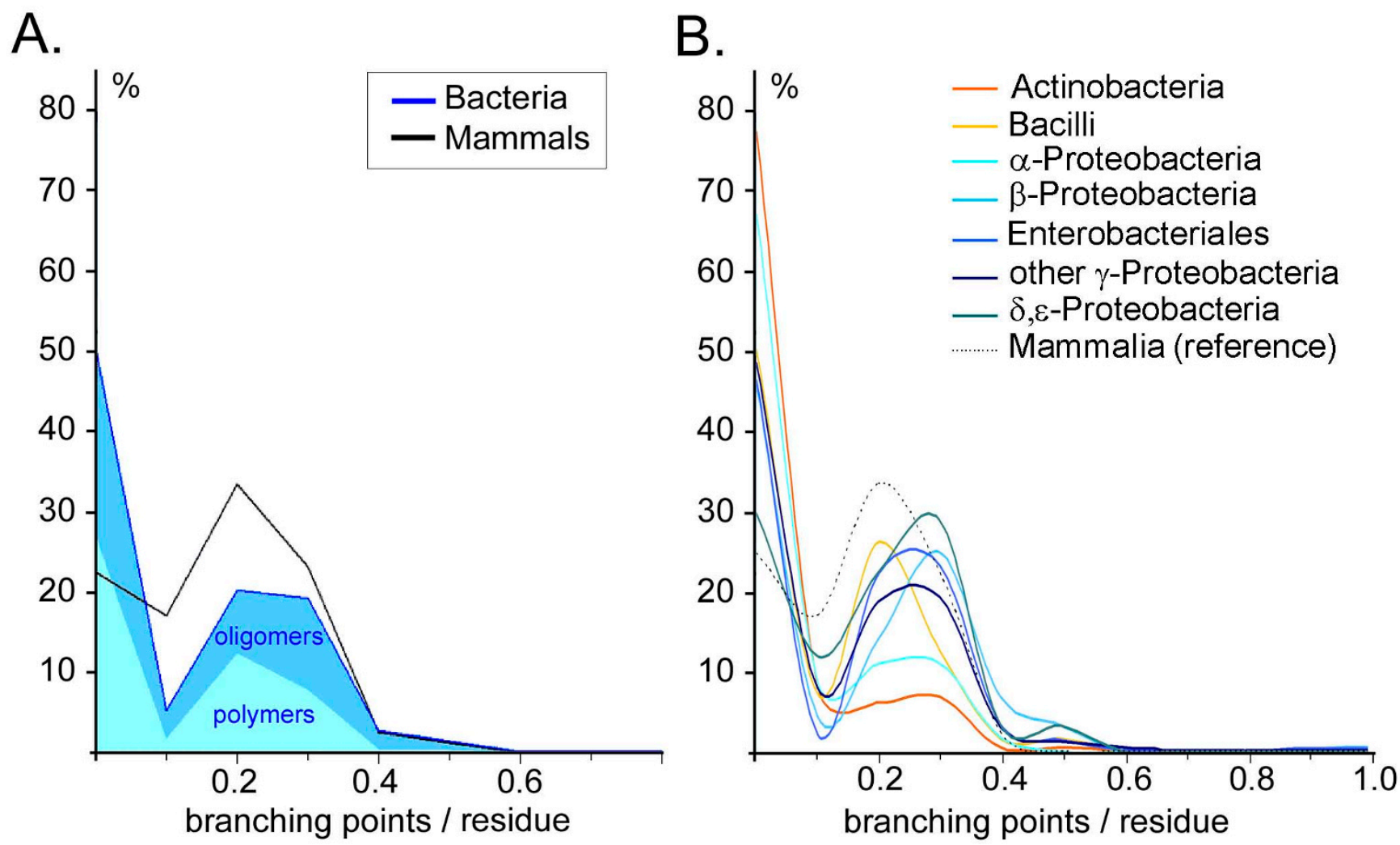

Figure 3

Branching index distribution. The normalized frequency distributions for the number of branching points per residue are shown for the carbohydrate sequence units of taxonomy Set I (A) and Set 2 (B), analogous to the graphs in Fig. 2.

COSCIENCES.de database a total of 35 different monosaccharides were found for mammalian carbohydrates, according to the nomenclature used in the original databases (Table 3). This degree of diversity is at first glance puzzling, in view of the common notion that mammalian carbohydrates are built up of 10 "classical" monosaccharides (Glc, Gal, GlcNAc, GalNAc, Man, GlcA, Fuc, Neu, IdoA, Xyl) [1]. However, the variety of monosaccharides defined in the primary databases is higher due to (a) residues being specified with unknown anomer or ring type definitions, (b) analytical artifacts from the structure elucidation process (alditols, double bonds), or (c) secondary modifications such as sulfation. Furthermore, carbohydrate sequence databases are not error-free and suffer from incorrect structure elucidations and curation mistakes. Since the existing databases generally use freetext identifiers for the monosaccharides, it was helpful to translate all structural database entries into a machinereadable notation called GlycoCT [16]. Using structural filters based on this notation we were able to significantly reduce the fuzziness introduced by the lack of strictness in the definitions of the original sequences (Table 3). During the analysis we excluded manually common artifacts caused by analytical procedures and entries with undefined absolute or anomeric configuration or ring type.

To minimize the influence of errors and artifacts on the statistics of Table 3, a threshold for the occurrence of monosaccharides, basetypes and basic entities was set at 10 for mammals and 2 for bacteria. This means that a given residue type was included in the statistics only when its number of occurrences exceeded the defined threshold. A relatively low threshold was chosen for bacteria because, in contrast to mammals, bacteria are known to produce a great variety of unique monosaccharide residues with low occurrence. When the threshold for bacteria was reduced from 2 to 0 , the diversity of detected residues increased by about 25\%. A complete list of monosaccharide residues and basetypes found for each taxonomic group is available in GlycoCT nomenclature in the additional material [see Additional files 2 (tables a-i) and 3].

For mammals the analysis returned 18 occurrences of DFuc as a basic entity. However, this residue was excluded from Table 3 because all carbohydrates in GLYCOSCIENCES.de which are specified to contain D-Fucose 


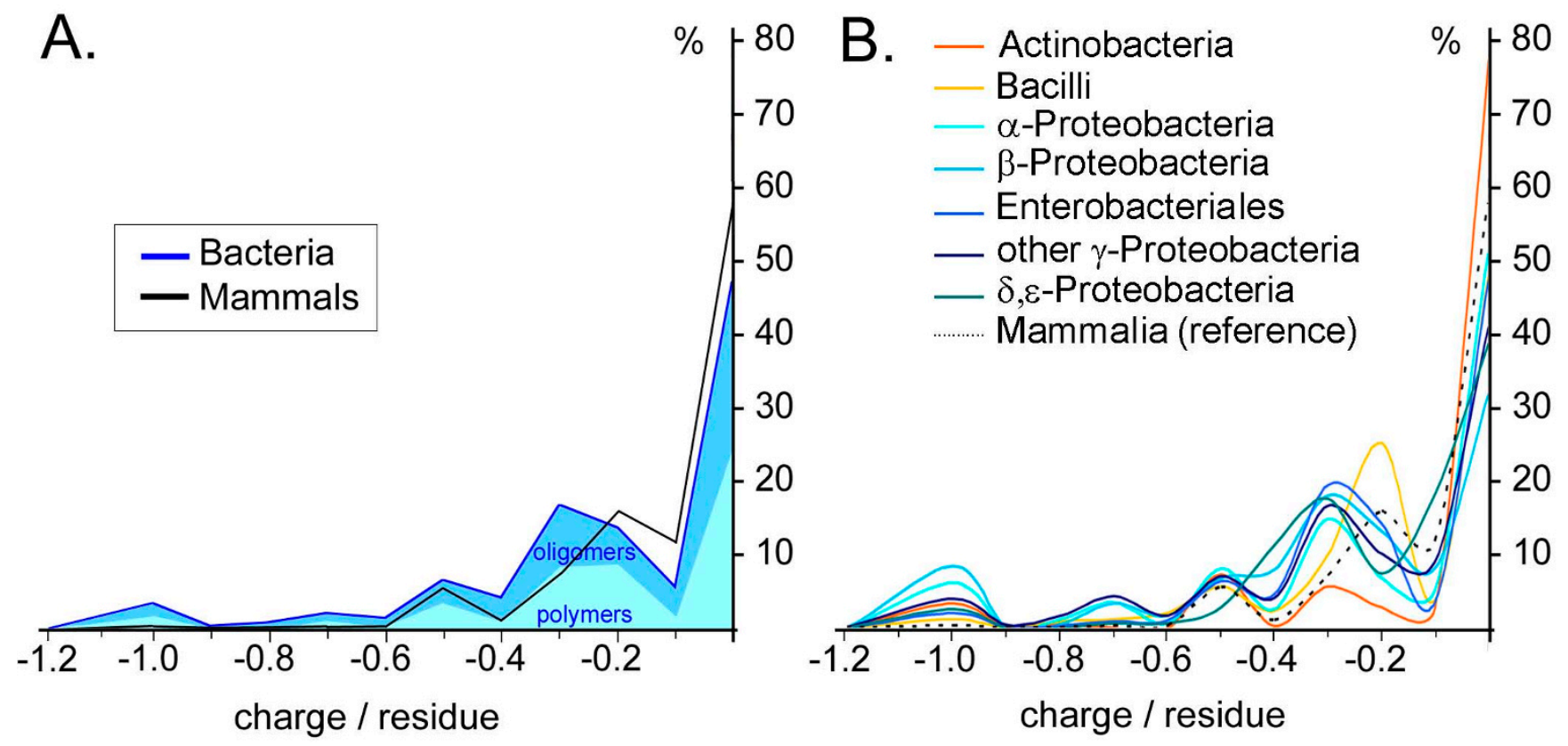

\section{Figure 4}

Mean charge density distribution. Normalized frequency distributions for the maximum possible mean charge density per residue are plotted for the carbohydrate sequence units of taxonomy Set I (A) and Set 2 (B), analogous to the graphs in Fig. 3.

originate from old publications in which the absolute configuration of Fuc was not specified. The occurrence of D-Fuc in mammalian carbohydrate records can be regarded as a data translation error since there is no evidence for a mammalian enzyme which synthesizes D-Fuc.

The 35 monosaccharides with the highest occurrence within taxonomy Set 1 are shown schematically in Fig. 5. Of them, all 17 monosaccharides that were found in mammalian carbohydrates are also found in the bacterial world. Rhamnose, L-glycero- $\alpha$-D-manno-Heptose, $\alpha$-DGalacturonic acid and $\alpha$-Kdo are the most frequent monosaccharides that are unique to bacteria and, except for
Rhamnose, are preferably located in the core portions of bacterial saccharides, in accordance with the typical lipopolysaccharide (LPS) structure of Gram-negative bacteria, the classes which dominate in this analysis (Table 2).

A more detailed analysis of monosaccharide residues in the bacterial taxonomy groups of Set 2 is shown in Fig. 6. Kdo and L-glycero-D-manno-Heptose are confined to Gramnegative bacteria, whereas Gram-positive bacteria seem to have an excess of arabinoses and methylated hexoses.

Table 3: Diversity of monosaccharides, basetypes and basic entities for various taxonomic groups.

\begin{tabular}{|c|c|c|c|}
\hline Group & Monosaccharides & Basetypes & Basic entities \\
\hline Mammalia & 35 & 14 & $10 *$ \\
\hline Bacteria & 551 & 143 & 123 \\
\hline Actinobacteria & 100 & 48 & 33 \\
\hline Bacilli & 100 & 41 & 34 \\
\hline$\alpha$-Proteobacteria & 68 & 34 & 26 \\
\hline$\beta$-Proteobacteria & 69 & 33 & 32 \\
\hline Enterobacteriales ( $\gamma$-Proteobacteria) & 243 & 76 & 65 \\
\hline other $\gamma$-Proteobacteria & 203 & 70 & $76 * *$ \\
\hline$\delta / \varepsilon-$ Proteobacteria & 52 & 35 & 32 \\
\hline
\end{tabular}

The total number of different monosaccharides, basetypes, and basic entities are listed for each group in taxonomy Set I and Set 2 . When counting in the combined databases, an occurrence threshold of 10 for mammals and 2 for bacteria was employed.

* The basic entity D-Fuc was excluded from Mammalia for reasons given in the text.

** In this case there are more basic entities compared to basetypes because amino derivatives are included in the basic entities. 


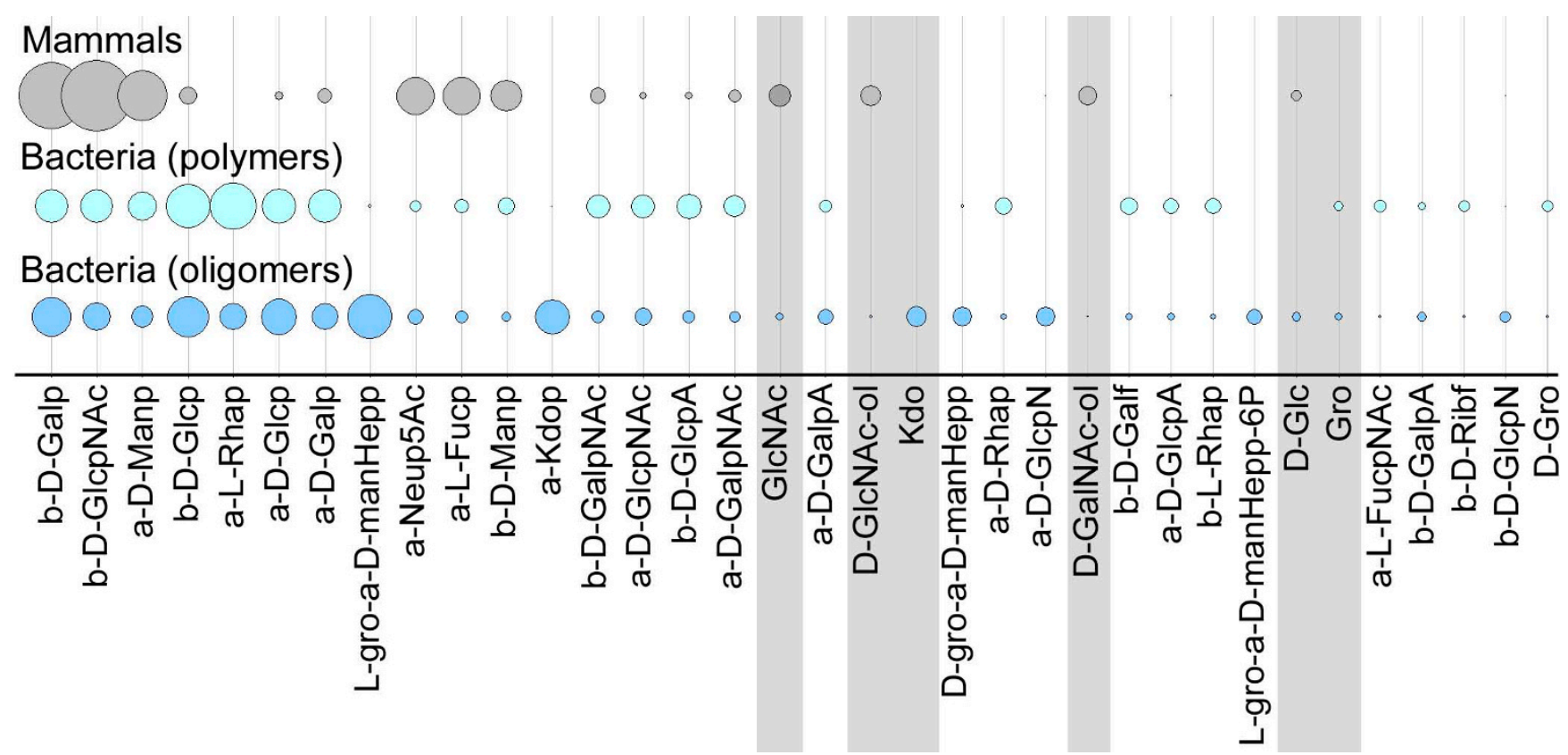

\section{Figure 5}

Diversity of monosaccharides in bacteria and mammals. For taxonomy Set I the 35 most abundant monosaccharide residues are listed from left to right in decreasing order of their total abundance across all taxonomic groups. The three rows of circles correspond to the three groups: mammals (gray), bacteria (polymers, cyan), bacteria (oligomers, blue), as defined in Table I. Circle areas reflect the relative abundance of a monosaccharide residue within each group (monosaccharide count/ total residue count per taxonomic group). Residues that are the result of analytical artifacts or incomplete structure elucidation (hexosamine alditols, glycerol, D-Glc, etc.) are highlighted with gray bars. In this and subsequent Figures the anomeric designators $\alpha$ and $\beta$ are written as a and $b$, the ring type designators $p$ and $f$ are shown as $p$ and $f$.

Generally, monosaccharides that are unique to bacteria are of special interest as potential immunogenic targets. Existing vaccines frequently take advantage of the unique saccharides in the complex carbohydrates located on the surface of bacteria [11]. Fig. 7 presents the unique monosaccharides (see definition of unique in the Figure legend) found for the bacterial and mammalian groups. Due to the greater diversity of bacterial monosaccharides, many carbohydrates unique to the bacterial world were found (especially for Gram-positive bacteria), whereas only two mammalian monosaccharides [ $\alpha-N$-Glycoloylneuraminic acid ( $\alpha$-Neu5Gc) and $\beta$-D-N-acetylglucosamine-6-O-sulfate ( $\beta$-D-GlcpNAc-6S)] appear to have no counterpart in the bacterial world. It is known that neuraminic acid derivatives are typically found at the terminal positions of mammalian glycoconjugates, being mediators for cell-cell interaction or receptors for pathogens [17]. The presence of exposed $\alpha$-Neu5Ac residues in bacteria may be an evolutionary advantage through which bacteria mask themselves to the host immune system.

Attention should be paid to the distribution of monosaccharides at the terminal positions of oligomers and side chains of polymers. In higher organisms such residues are optimally positioned to mediate recognition by endogenous carbohydrate-binding proteins [9]. According to our findings bacterial carbohydrates often have glucose residues at the nonreducing ends, in contrast to mammalian glycans (data not shown). This may be the result of the evolutionary adaptation of bacteria since exposed terminal glucose residues are important for the adherence of bacteria and entry into host epithelial cells, as demonstrated for Salmonella and Pseudomonas [18].

Fig. 8 demonstrates that more than $70 \%$ of the monosaccharides in every taxonomic group are reported to be in the pyranose form, with most groups even reaching $90 \%$. An interesting finding is that more than $50 \%$ of all furanose residues found in bacteria are in the 395 glycan sequences of the class Actinobacteria (cf. area of bars in Fig. $8 B$ ). Nearly $20 \%$ of all residues in Actinobacteria glycans are in the furanose form, compared to $<4 \%$ for all other bacterial groups studied. A high proportion of furanose residues has also been found for plants (data not shown). In the majority of cases where linear forms or rings of unknown size are found, they can be explained as artifacts of the structure elucidation process, especially when present at the reducing end. However, linear monosaccha- 
A.

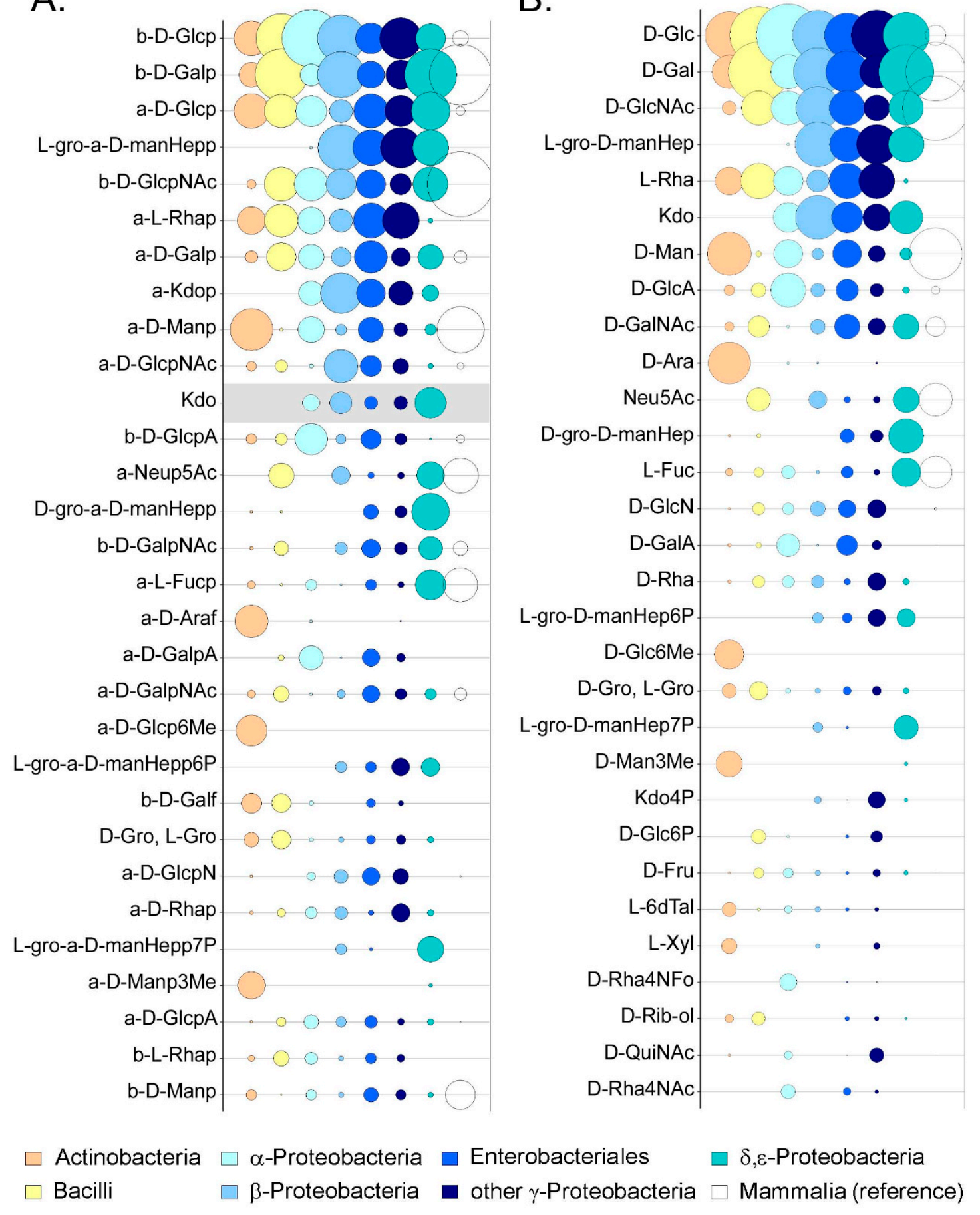

\section{Figure 6}

The most abundant monosaccharides in bacteria. Circle areas reflect relative abundances of the 30 most common monosaccharide residues $(\mathbf{A})$ or basic entities (B) for the carbohydrates found in the BCSDB for each of the bacterial taxonomy groups of Set 2. Within each group the abundances are normalized to the total number of residues per group. The color code (see legend at bottom) is the same as in Figs. 2-4; for comparison, the open circles represent data for mammals. The residues are sorted from top to bottom in order of decreasing total abundance in bacteria (order differs from Fig. 5). The residue $\mathrm{Kdo}$ (without anomeric configuration) results from analytical artifacts and is highlighted in gray. For the basic entities defined in $\mathbf{B}$, no distinction is made between anomeric configurations and ring types. 

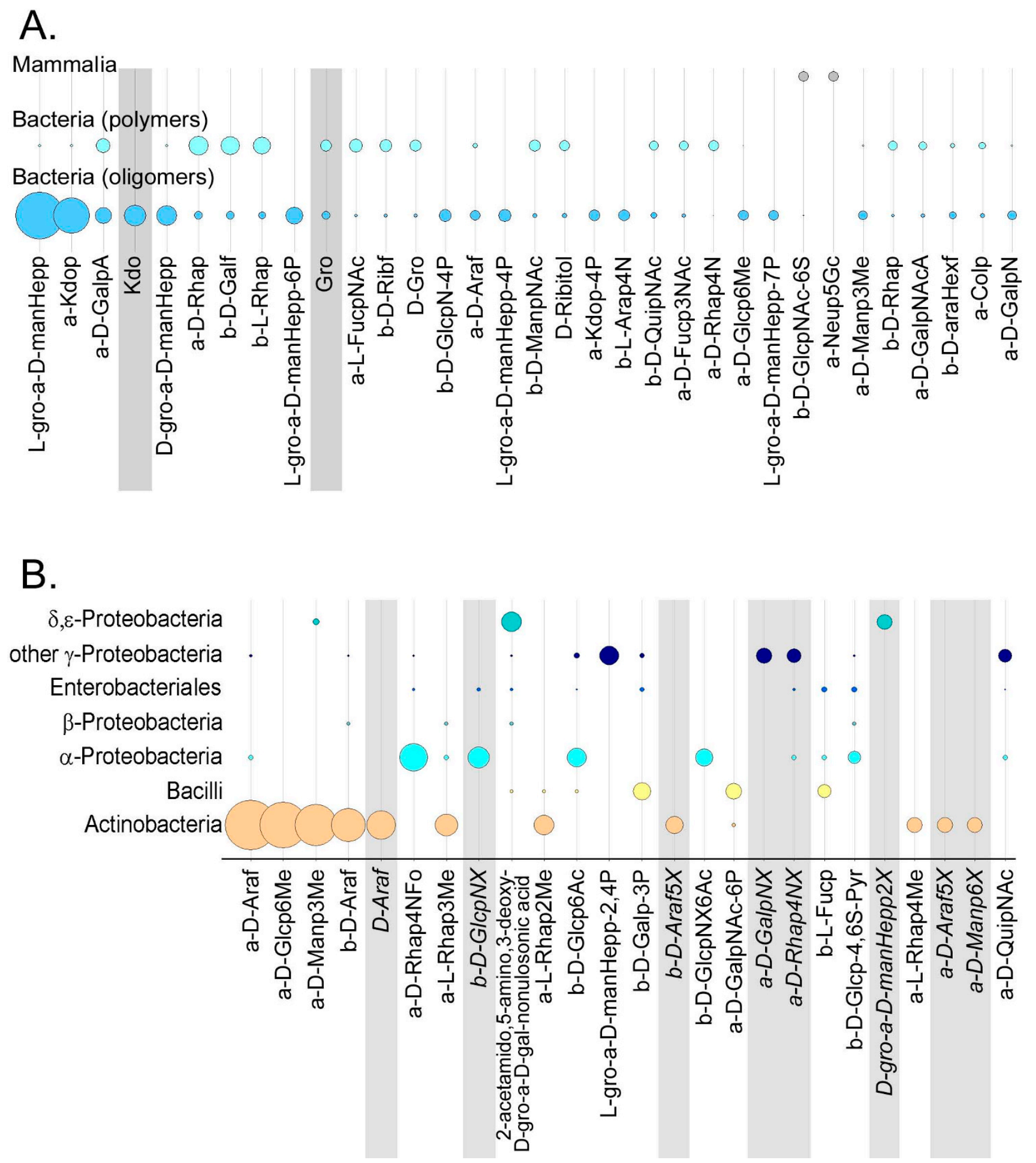

\section{Figure 7}

The most abundant unique monosaccharides. Circle areas reflect relative abundances within a taxonomic group for those unique monosaccharide residues which appear exclusively, or nearly so, in a single taxonomic group of Set I (A) or Set 2 (B). Uniqueness is defined here as: frequency in the selected group $>0.1 \%$, frequency in other groups $<0.1 \%$. Residues that result from analytical artifacts and those that are ambiguous due to incomplete structure elucidation are highlighted in gray. The symbol $X$ represents any substituent. 


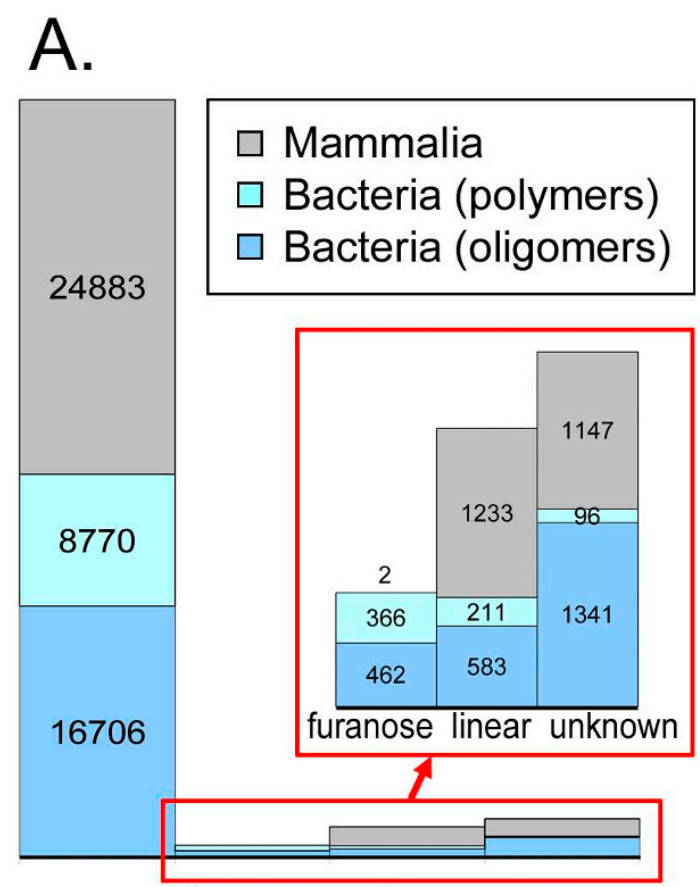

pyranose furanose linear unknown

B.

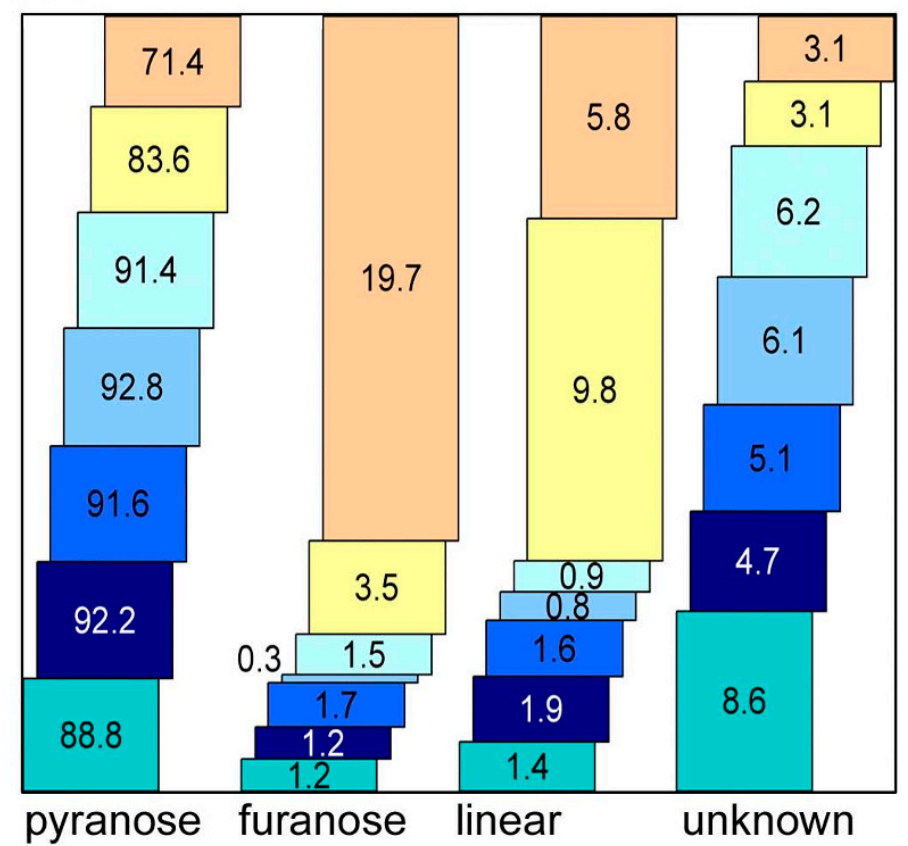

Actinobacteria

Bacilli

$\alpha-$ Proteobacteria

$\beta$-Proteobacteria

Figure 8

Monosaccharide ring type distributions. The distribution of residue ring type (pyranose, furanose, linear, unknown) is shown schematically for various taxonomic groups. A. For taxonomy Set I the areas of the colored bars are proportional to the absolute occurrences (numbers shown) of a given ring type in each taxonomic group. The vertical scale is expanded in the inset. B. For taxonomy Set 2 two different ways of viewing the data are presented. (I) For a given ring type the area or height of each colored bar in a stack represents the relative abundance (\%) of that ring type for each of the taxonomic groups, normalized to the total occurrence of that ring type across all groups (stack height $=100 \%$ for each ring type). Thus, the bar heights within a stack represent the distribution of a single ring type across all taxonomic groups. (2) The number labeled in each bar represents the frequency (in \%) of residues with the corresponding ring type within the bar's taxonomic group, normalized to the total number of residues for that group. The numbers sum horizontally to $100 \%$ for each taxonomic group (color) and, therefore, represent the distribution of the different ring types within an individual group.

rides are known to occur occasionally in bacterial carbohydrate sequences and are most prevalent in Bacilli and Actinobacteria (Fig. 8B).

\section{Monosaccharide modifications}

Part of the diversity of monosaccharides can be found in their modifications (Fig. 9). For bacteria secondary modifications often play a role in the mediation of reactivity and lability to various environmental conditions such as $\mathrm{pH}$. The $N$-acetylamino group is the most common substituent for carbohydrates in mammals (ca. $45 \%$ of all res- idues) and in most bacteria classes (ca. 18-21\% of all residues), except for $\alpha$-Proteobacteria (11\%) and Actinobacteria $(4.5 \%)$. Acetylation of amino groups plays a key role in regulating the ability of amino sugars to form hydrogen bonds and to bear charge [19].

O-methylation is the most frequent modification for Actinobacteria (ca. 23\% of all residues, mainly at $\mathrm{O} 6$ of glucose) but occurs with a frequency of $<5 \%$ in other bacteria classes and is essentially absent in mammals. 


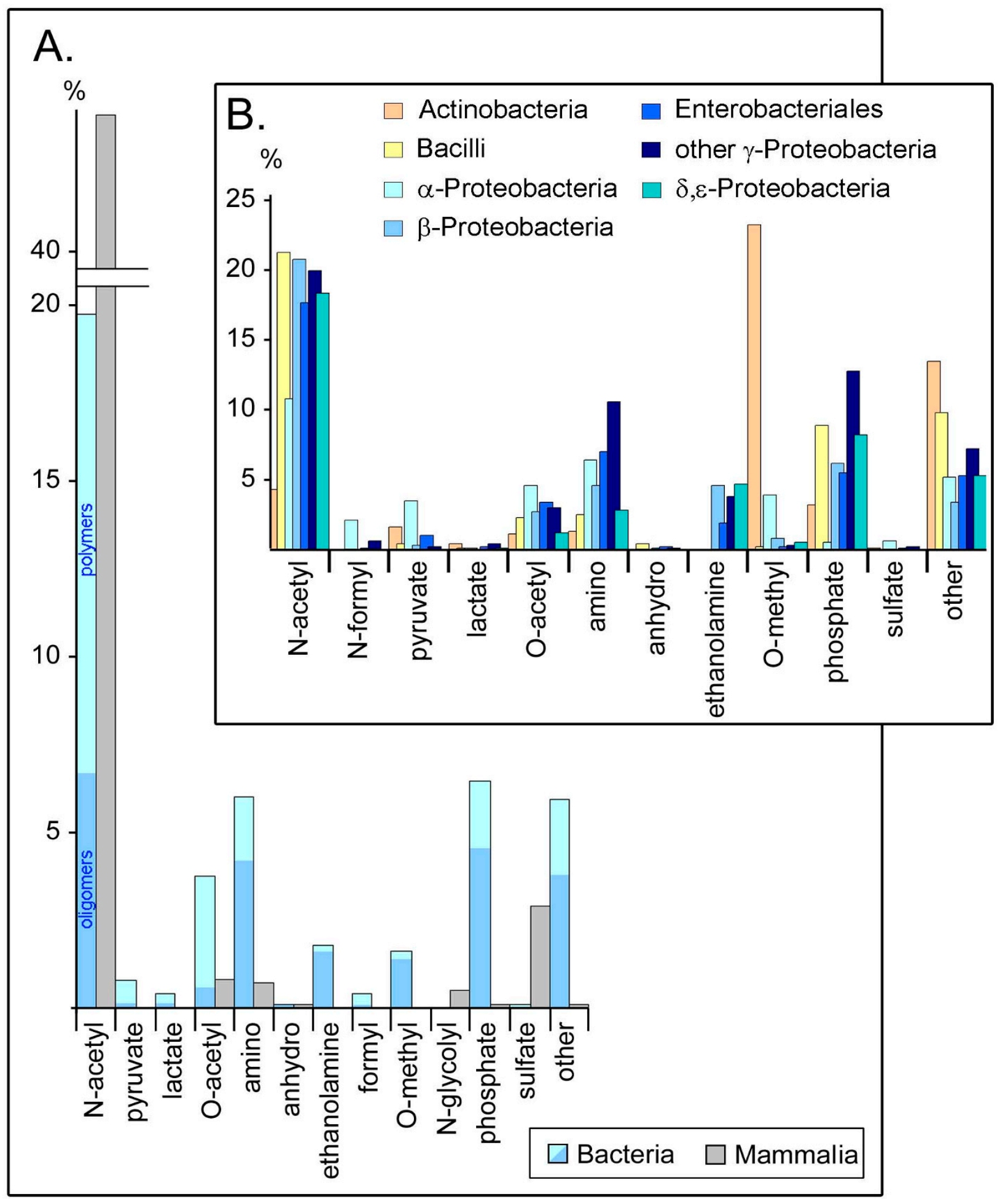

Figure 9

Distribution of monosaccharide modifications. Frequency distributions (in \%) for secondary modifications of monosaccharide residues are shown for taxonomy Set I $(\mathbf{A})$ and Set $2(\mathbf{B})$, normalized to the total number of carbohydrate residues within each taxonomic group. In $\mathbf{A}$ the bars for bacterial oligomers (blue) and polymers (cyan) are stacked to give the cumulative values for all bacteria studied. 
O-acetyl, amino, or phosphate substituents are also much more prevalent for bacteria (4-7\%) than for mammals (< $1 \%$ ), and the $O$-acetylation pattern is often different for different cultures of a single bacteria strain. $O$-acetyl groups mask the protective epitopes for bacteria through steric hindrance or altered conformations, as shown for Meningococci [20].

Amino sugars with free aminogroups are present in about $7 \%$ of bacterial carbohydrate residues compared to ca. $1 \%$ for mammals and feature a positively charged $-\mathrm{NH}_{3}{ }^{+}$substituent at neutral $\mathrm{pH}$. The occurrence of these residues in the bacterial cell wall affects hydrophobicity and makes bacteria resistant to the lysozyme of the host, as has been demonstrated for glucosamine in Gram-positive bacteria [21]. Several secondary modifications appear to be unique for bacterial carbohydrates (pyruvate, lactate, ethanolamine, O-methyl and formyl) while sulfation or $\mathrm{N}$ glycolyl substitution occurs primarily in mammals. Finally, about $7 \%$ of bacterial residues have modifications listed under the category "other" in Fig. 9A, with Actinobacteria and Bacilli showing the highest frequencies (Fig. 9B).

\section{Disaccharide fragment patterns in bacteria and mammals} The topological characteristics of glycan architecture can be described by statistics which document the frequency distributions for specific neighboring pairs of monosaccharides connected either with any type of linkage (monosaccharide pair analysis) or via specific linkage positions (disaccharide pattern analysis). The matrix diagram in Fig. 10 illustrates the statistics of linked monosaccharide pairs for bacteria. Here the frequencies of any type of glycosidic linkage between the 20 most common donor and acceptor residues are shown. The areas of the circles plotted at the coordinates for a given pair represent its relative abundance within a given bacterial taxonomic group. Note that not all possible monosaccharide pairs are actually found in the natural sequences registered in the database (missing circles). Some combinations are exclusive for Grampositive bacteria, e.g. those involving $\alpha$-D-Araf or $\alpha$-DGlcp6Me in Actinobacteria while others may exhibit similar or widely differing abundances across the taxonomic groups. The high abundances found along the diagonal of the matrix stem from homopolymeric subsequences which are frequent in bacteria. Note that the results for "Kdo" (without anomeric configuration) originate from analytical artifacts. Detailed results for a total of 676 pairs in bacterial and mammalian carbohydrates are summarized in the additional material section [see Additional file 4].

In order to describe carbohydrate sequences at a higher level of complexity, we need to consider not only the identities of the linked monosaccharides but also the link- age configuration. All free hydroxyl groups on each acceptor monosaccharide are potential sites of glycosyltransferase reactions. Therefore, we define the child (donor) to parent (acceptor) connection in terms of the directed glycosylation linkage pattern, analogous to reaction patterns described elsewhere [22]. Thus, the descriptor "a1-4", for example, indicates that an alpha anomeric $\mathrm{O} 1$ of the donor is linked to $\mathrm{C} 4$ of the acceptor. The statistics of linkage patterns provide a direct description of the expression and activity of glycosyltransferases and the carbohydrate structure repetoire in an organism or taxonomic group. Such statistics have been employed successfully for a variety of bioinformatic tasks with the glycome, e.g. matrix generation [23] and pattern detection [24]. This kind of information is also valuable for recognizing both unique and common linkages and can serve as a basis for a deeper understanding of the immunogenicity of bacterial carbohydrates and for designing targeted vaccines.

The analysis summarized in Fig. 11 demonstrates that the most prevalent linkages in mammals are D-Gal, D-Man and D-GlcNAc as $\beta 1-4$ donors to D-GlcNAc; D-GlcNAc as $\beta 1-2$ donor to D-Man; D-GlcNAc as $\beta 1-3$ donor to D-Gal; and D-Man disaccharides with $\alpha 1-3$ or $\alpha 1-6$ linkages. These monosaccharides are sufficient to build up the common $\mathrm{N}$ - and $\mathrm{O}$-glycan structures which dominate the mammalian database.

A complete list of mammalian disaccharide fragments found in GLYCOSCIENCES.de is available [see Additional file 5], where the data are encoded to illustrate the differences between our database findings and the data from Ohtsubo \& Marth [2]. Three disaccharides reported in [2], namely D-Glc $(\alpha 1-2)$ D-Gal, D-GlcA( $\beta 1-4)$ D-Gal and D$\operatorname{GlcNAc}(\alpha 1-6)$ D-GlcNAc, are absent from our databases. On the other hand, many existing mammalian disaccharides have not been mentioned by Ohtsubo \& Marth, among them reasonably abundant ones such as D-GlcNAc $(\beta 1-3)$ D-GalNAc, D-GlcNAc( $\beta 1-4)$ D-Man, D-GlcNAc $(\beta 1-6)$ D-Man, and D-GlcNS $(\alpha 1-4)$ L-IdoA. The last disaccharide listed is present in more than 500 records of GLYCOSCIENCES.DE for human carbohydrates and reported in the literature in association with Sandhoff's Disease [25].

The disaccharide ensemble or total count of unique linkages is considerably larger for most of the bacterial classes compared to mammals, as shown in Table 4 . For this analysis we have taken a total of 24613 bacterial and 23883 mammalian disaccharide fragments into account.

The abundance data for the more frequently occurring disaccharide linkage patterns found in mammals and/or bacteria are presented in Table 5 (detected in the combined 


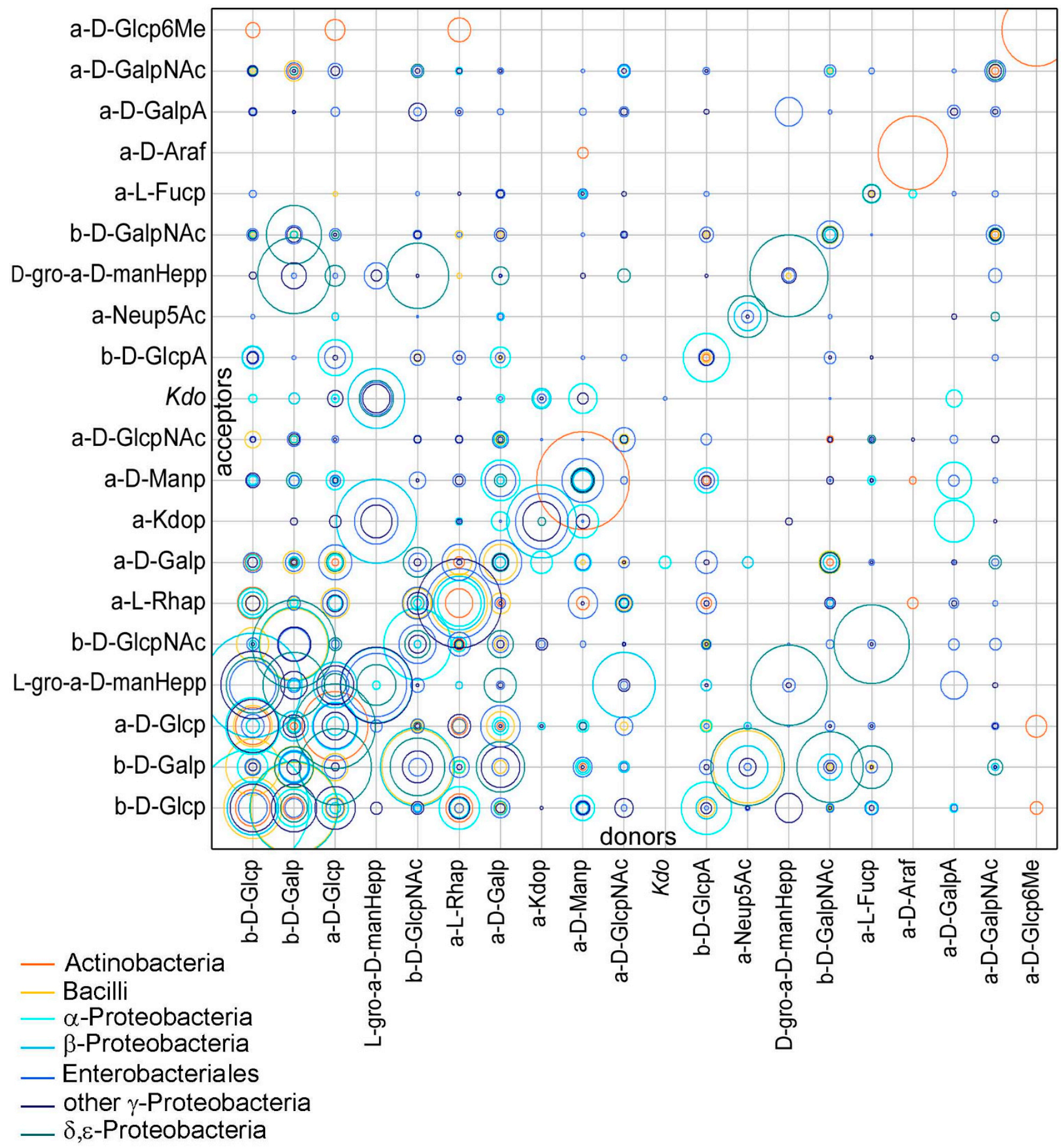

Figure 10

Distribution of monosaccharide pairs in various bacterial groups. For taxonomy Set 2 the matrix presents relative abundance data for monosaccharide residue pairs of all linkage types, involving the 20 most common residues serving as donor (children) or acceptor (parent). Each circle area reflects the relative abundance of a given donor-acceptor pair (matrix coordinates) within the corresponding taxonomic group, normalized to the total number of pairs within that group. 


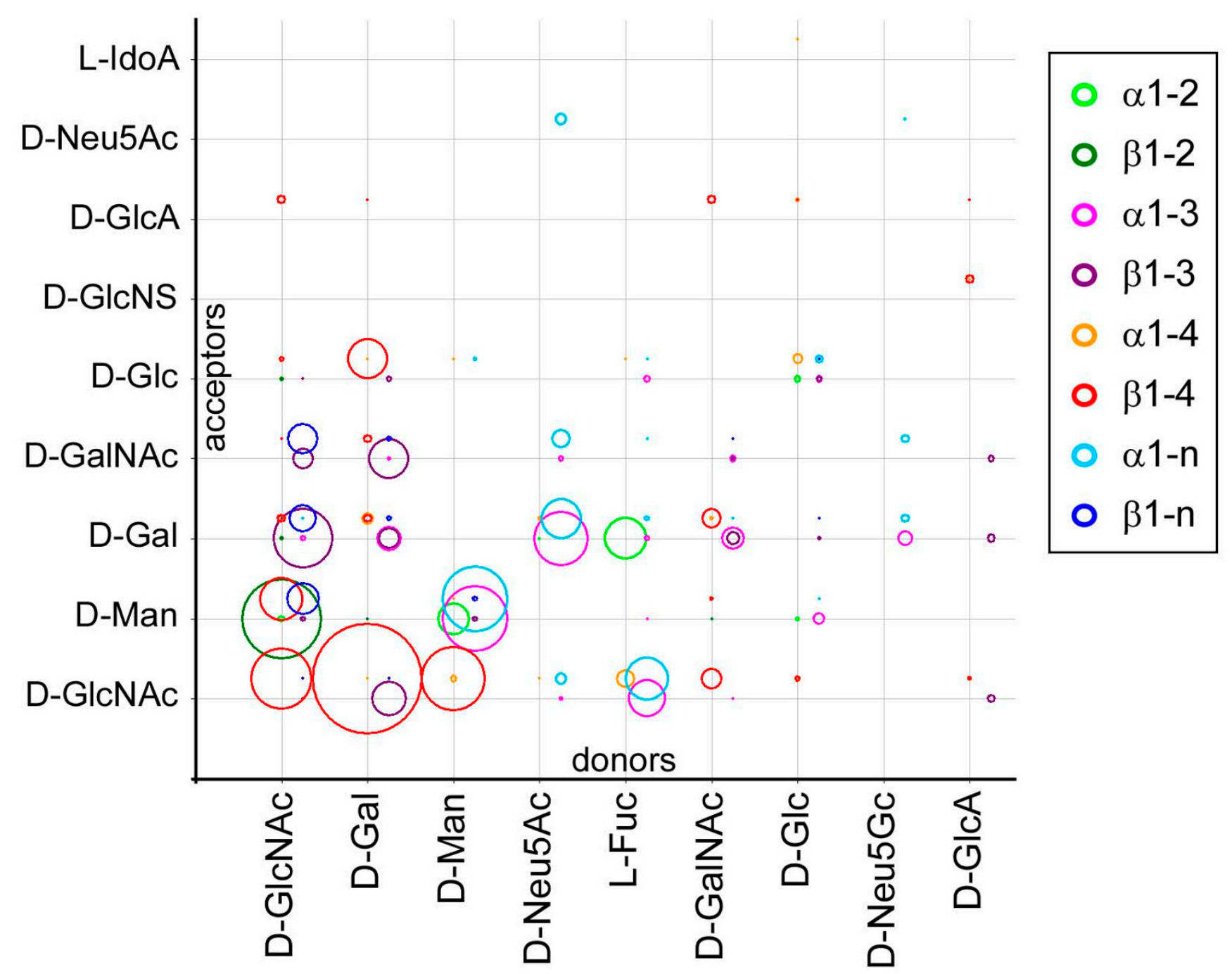

\section{Figure I I}

Glycosidic linkages in mammalian carbohydrates. Frequency distribution of specific disaccharide linkages in mammalian carbohydrates. Plotted circle areas represent the relative frequencies for disaccharides formed from the 9 most common donors (children) and 9 most common acceptors (parents) in a defined glycosidic linkage (color code in legend). The areas of the circles are proportional to the relative abundances of disaccharide pairs, normalized to the total number of specific disaccharide pairs. The linkage codes $\alpha \mid-n$ and $\beta I-n$ correspond to a linkage to any exocyclic carbon at the acceptor, e.g. C6 in hexopyranoses. For donor residues in keto form the linkage is at the anomeric carbon $\mathrm{C} 2$ instead of $\mathrm{Cl}$. For better visualization some of the circles for a given linkage are offset somewhat from the matrix coordinate corresponding to a given linkage type.

database using an abundance threshold $=0.1 \%$ for either mammals or bacteria). More comprehensive data (with lower threshold) are presented in the additional material [see Additional file 6]. In Table 5 there are 26 disaccharide linkages listed which are found only in bacteria. The four most frequent of these, with abundances of $0.40-0.68 \%$ (underlined in Table 5) are D-Glc $(\alpha 1-4)$ D-Gal, DGal( $\alpha 1-2)$ D-Gal, D-Glc(b1-4) D-Gal, and D-Gal( $\alpha 1-2)$ D-Man. In Table 5 there are five disaccharide types which occur in mammals only, and the most abundant of these (underlined in Table 5) involve (a) $\alpha 2-6$ linkages from a neuraminic acid (Neu5Ac or Neu5Gc) to D-GalNAc or (b) a1-4 linkages from a uronic acid (GlcA, IdoA, or $\Delta$ GlcA) to D-GlcN-sulfate.

The abundances of the most common bacterial disaccharide fragments are shown schematically in Fig. 12, where separate diagrams are presented for oligomers (A) and polymers (B) using the same color-coded linkage scheme as in Fig. 11. The residue names are sorted according to their abundance either as donors (children) or acceptors (parents). The highest abundances in bacterial oligomers 
Table 4: Diversity of disaccharide linkages found in various taxonomic groups.

\begin{tabular}{lc}
\hline Group & Disaccharide Linkages \\
\hline Actinobacteria & 364 \\
Bacilli & 526 \\
$\alpha-P r o t e o b a c t e r i a$ & 319 \\
$\beta$-Proteobacteria & 356 \\
Enterobacteriales ( $\gamma$-Proteobacteria) & 1570 \\
other $\gamma$-Proteobacteria & 1148 \\
S/E-Proteobacteria & 218 \\
Mammalia & 488
\end{tabular}

Total count of all unique disaccharide linkages per taxonomic group. Unknown anomeric and absolute configurations and fuzzily defined linkages are counted as distinct entities.

(Fig. 12A) are exhibited by the constituents of the bacterial lipopolysaccharide core region: L-gro-D-manHep$\rightarrow$ Kdo and L-gro-D-manHep $\rightarrow$ L-gro-D-manHep. High abundances for L-Rha $\rightarrow$ L-Rha, D-Man $\rightarrow$ D-Man, D-Gal $\rightarrow$ DGal and D-Glc $\rightarrow$ D-Glc in Fig. 12B arise from the homopolymeric regions prevalent in bacterial polymers.

A potential application of the information and methods outlined here is the design and validation of carbohydrate vaccines against bacterial pathogens. Carbohydrate-based vaccines against Haemophilus influenzae Type b, Neisseria meningitidis and Streptococcus pneumoniae have already been licensed, and many similar products are in various stages of development. For example, the disaccharides DGlc $(\alpha 1-2) D-G a l$ and D-Glc $(\beta 1-4) D-G a l$ are not present in mammalian organisms accordingly to our analysis and are both constituents of the capsular polysaccharides of Salmonella pneumonia, which were shown to be target candidates for vaccine development [26].

\section{Conclusion}

In this study we combined the $\mathrm{BCSDB}$, the largest available bacterial carbohydrate database, with the GLYCOSCIENCES.de database to obtain a set of 13775 nonredundant glycan/taxon pairs (carbohydrate sequences with a defined taxonomy), of which 6098 were assigned to Bacteria and 4739 to Mammalia. The representative statistical analyses presented here reveal the basic principles of carbohydrate architecture in bacteria vs. mammals. The major monosaccharides which characterize different branches of the tree of life were extracted from the database and are in accordance with the published literature. Several monosaccharides unique to certain subclasses of bacteria were identified and could prove useful as molecular markers for these classes. Similarly, a variety of structural modifications of monosaccharides have been detected, and many of these are characteristic in that they may be either highly abundant or totally absent in individual taxonomic classes.
A linkage analysis was performed for all disaccharide fragments of bacterial and mammalian glycans and revealed that there are a number of abundant linkages as well as nonexistent linkages which may be useful for characterizing the various taxonomic groups. Through a comparison of the disaccharide linkage ensembles or spaces for bacteria and mammals, one obtains an overview of those glycosyltransferase activities which are common to both classes and those which appear to be unique for mammals or bacteria or even for specific bacteria subclasses. Thus, differential cross-species expression analysis is possible and may ultimately provide a deeper understanding of immunogenic patterns present in pathogenic bacteria.

\section{Methods}

The analyzed sequences were obtained from the metadatabase GlycomeDB [27], which contains all sequences from the Bacterial Carbohydrate Structure DataBase (BCSDB) [12] and the GLYCOSCIENCES.de portal [13] in a harmonized format. With the help of the NCBI taxonomy database [15], subsets of these databases were taken and further analyzed using routines implemented in JAVA. The results of the analytic routines were stored in a PostgreSQL 8.2 database. Additional analytical procedures were implemented in PHP, which finally generated Microsoft Excel tables used for further analysis and graphical visualization.

\section{Definition of terms}

According to IUPAC nomenclature a monosaccharide is a poly(hydroxy) aldehyde or ketone with three or more carbon atoms (triose, tetrose, etc.); the term denotes a single structural component without glycosidic linkages and includes a variety of derivatives such as amino, deoxy or carboxy forms. Oligosaccharides are compounds in which monosaccharides and their derivatives are coupled in a precisely defined manner via glycosidic linkages. The term polysaccharide generally refers to oligosaccharides with a large or undefined number of monosaccharide residues. The term carbohydrate includes all mono-, oligo- or polysaccharides and molecules derived from monosaccharides modified by reduction, oxidation, or substitution. The term glycan is frequently used to refer to any saccharide component of a glycoconjugate, such as a glycoprotein or glycolipid, even when the chain length is short. A glycoconjugate is formed by a covalent linkage between a glycan and a nonglycan entity. Polysaccharide may be used to refer to polymers with glycosidic and/or phosphodiester linkages (such as teichoic acids). The carbohydrate databases used in this study may contain any of the compounds described above but do not contain DNAs or RNAs.

In this study we used the following definitions: 
Table 5: Identity and abundances of mammalian and bacterial disaccharides.

\begin{tabular}{|c|c|c|c|c|c|c|c|c|c|c|}
\hline \multirow[b]{2}{*}{$\begin{array}{l}\text { Donors } \\
\text { (children) }\end{array}$} & \multicolumn{10}{|c|}{ Acceptors (parents) } \\
\hline & Fuc & Gal & GalNAc & Glc & GIcNAc & $\begin{array}{c}\text { GlcA, IdoA or } \\
\Delta \text { GIcA }\end{array}$ & Man & $\begin{array}{l}\text { Neu5Ac or } \\
\text { Neu5Gc }\end{array}$ & Xyl & GlcNS \\
\hline Fuc & $\mathrm{al}-3(0.01)[0.15]$ & al-2 (2.68) $[0.18]$ & & & $\begin{array}{l}\mathrm{al}-3(2.00)[0.32] \\
\mathrm{al}-4(0.54)[0.09] \\
\mathrm{al}-6(2.84)[0.01]\end{array}$ & & & & & \\
\hline Gal & & $\begin{array}{c}\text { al-3 }(0.92)[0.60] \\
\text { al-4 (0.22) [0.54] } \\
\text { al-6 (0.02) [0.25] } \\
\text { bl-3 }(0.70)[0.80] \\
\text { bl-4 (0.1) }[0.34] \\
\text { bl-6 }(0.05)[0.42] \\
\text { al-2 }[0.52]\end{array}$ & $\begin{array}{l}\text { al-3 }(0.02)[0.12] \\
\text { bl-3 (2.4I) [0.54] } \\
\text { bl-4 (0.1I) [0.1I }]\end{array}$ & $\begin{array}{c}\text { al-3 }(0.00)[0.32] \\
\text { al-6 }(0.00)[0.49] \\
\mathrm{bl}-3(0.04)[0.18] \\
\mathrm{bl}-4(2.5 \mathrm{I})[2.32] \\
\mathrm{bl}-6(0.00)[0.18] \\
\mathrm{al}-2[0.25]\end{array}$ & $\begin{array}{l}\text { al-3 }(0.00)[0.26] \\
\text { bl-3 }(1.86)[0.52] \\
\text { bl-4 (18.06) }[1.68]\end{array}$ & & $\begin{array}{l}\text { al-2 }[0.40] \\
\text { al-3 }[0.10] \\
\text { al-6[0.12] }\end{array}$ & & $\mathrm{bl}-4(0.1 \mathrm{l})[0.00]$ & \\
\hline GalNAc & & $\begin{array}{l}\text { al-3 }(0.80)[0.05] \\
\text { bl-3 }(0.30)[0.44] \\
\text { bl-4 (0.61) [0.36] }\end{array}$ & $\begin{array}{c}\mathrm{al}-3(0.07)[0.32] \\
\mathrm{bl}-3(0.03)[0.10] \\
\mathrm{al}-4[0.14] \\
\mathrm{bl}-4[0.20]\end{array}$ & & $\mathrm{bl}-4(0.68)[0.02]$ & $\mathrm{bl}-4(0.12)[0.08]$ & & & & \\
\hline Glc & & 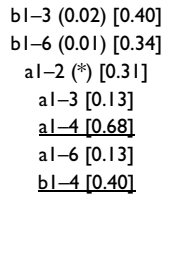 & $\begin{array}{l}\mathrm{al}-6[0.12] \\
\mathrm{bl}-3[0.18]\end{array}$ & $\begin{array}{c}\text { al-2 (0.06) [0.95] } \\
\text { al-3 }(0.08)[0.83] \\
\text { al-4 }(0.15)[0.39] \\
\text { al-6 }(0.10)[0.57] \\
\mathrm{bl}-3(0.05)[0.62] \\
\mathrm{bl}-4(0.00)[1.69] \\
\mathrm{bl}-6(0.01)[0.90] \\
\mathrm{al}-1[0.16] \\
\mathrm{bl}-2[0.33]\end{array}$ & $\begin{array}{l}\mathrm{bl}-3[0.11] \\
\mathrm{bl}-6[0.15]\end{array}$ & $\begin{array}{c}\text { al-4 }(0.03)[0.27] \\
\mathrm{bl}-4(0.01)[0.12] \\
\mathrm{bl}-3[0.10]\end{array}$ & $\begin{array}{l}\mathrm{al}-3(0.19)[0.19] \\
\mathrm{bl}-4(0.00)[0.14]\end{array}$ & & & \\
\hline GIcNAc & & $\begin{array}{l}\text { bl-3 (5.38) [1.64] } \\
\text { bl-4 }(0.13)[0.02] \\
\text { bl-6(1.17) }[0.04]\end{array}$ & $\begin{array}{l}\text { bl-3 }(0.68)[0.04] \\
\text { bl-6 (1.45) [0.00] }\end{array}$ & & $\begin{array}{c}\mathrm{bl}-3(0.0 \mathrm{l})[0.34] \\
\mathrm{bl}-4(5.6 \mathrm{I})[0.69] \\
\mathrm{bl}-6(0.0 \mathrm{I})[0.22] \\
\mathrm{bl}-2[0.15]\end{array}$ & $\begin{array}{l}\mathrm{al}-4(0.10)[0.05] \\
\mathrm{bl}-4(0.13)[0.13]\end{array}$ & $\begin{array}{l}\mathrm{bl}-2(9.46)[0.1 \mathrm{I}] \\
\mathrm{bl}-4(2.83)[0.05] \\
\mathrm{bl}-6(1.64)[0.02]\end{array}$ & & & \\
\hline $\begin{array}{l}\text { GlcA, IdoA } \\
\text { or } \triangle \text { GIcA }\end{array}$ & & bl-3 (0.13) [0.23] & $\mathrm{al}-3(0.14)[0.01]$ & $\mathrm{bl}-4[0.26]$ & $\begin{array}{l}\text { al-4 }(0.16)[0.01] \\
\text { bl-3 }(0.13)[0.14]\end{array}$ & $\begin{array}{c}\mathrm{bl}-4(0.0 \mathrm{l})[0.19] \\
\mathrm{bl}-3[0.13]\end{array}$ & $\begin{array}{l}\text { al-3 [0.22] } \\
\text { bl-2 [0.19] }\end{array}$ & & & $\frac{\mathrm{al}-4(0.44)}{\mathrm{bI}-4(0.10)}$ \\
\hline Man & & al-3 [0.22] & & al-3 $(0.00)[0.27]$ & b|-4 (6.22) [0.02] & & $\begin{array}{c}\text { al-2 (I.60) [0.92] } \\
\text { al-3 }(6.39)[0.41] \\
\text { al-4 (0.0I) [0.16] } \\
\text { al-6 }(6.35)[0.53] \\
\text { bl-4 [0.17] }\end{array}$ & & & \\
\hline $\begin{array}{l}\text { Neu5Ac or } \\
\text { Neu5Gc }\end{array}$ & & $\begin{array}{l}\text { a2-3 (4.85) [1.01] } \\
\text { a2-6 (2.64) [0.02] }\end{array}$ & $\underline{\mathrm{a} 2-6}(0.49)$ & & a2-6 $(0.22)$ & & & a2-8 (0.29) [0.12] & & \\
\hline Xyl & & & & & & & & & bl-4 (0.00) [0.10] & \\
\hline GIcNS & & & & & & al-4 (0.34) & & & & \\
\hline
\end{tabular}

Linkage types and relative abundances are shown as percentages (rounded to two decimals) of the total disaccharide fragment count for mammals (values in parentheses) or bacteria [values in brackets. Linkages are listed only for disaccharide fragments present at $>$
$0.1 \%$ in either taxonomy group. Values of 0.00 indicate the presence of a linkage at $<0.005 \%$; missing values indicate the absence of that linkage type in the corresponding taxonomy group. An entry $(*)$ denotes that the linkage was reported for mammals in 2 ] b but was not found in the databases. The percentages are cumulative for all ring types (including alditols), all absolute configurations and for all anomeric configurations of the acceptor. For linkages that occur only in mammals or only in bacteria, those with abundences of
$0.40 \%$ or more are underlined. 


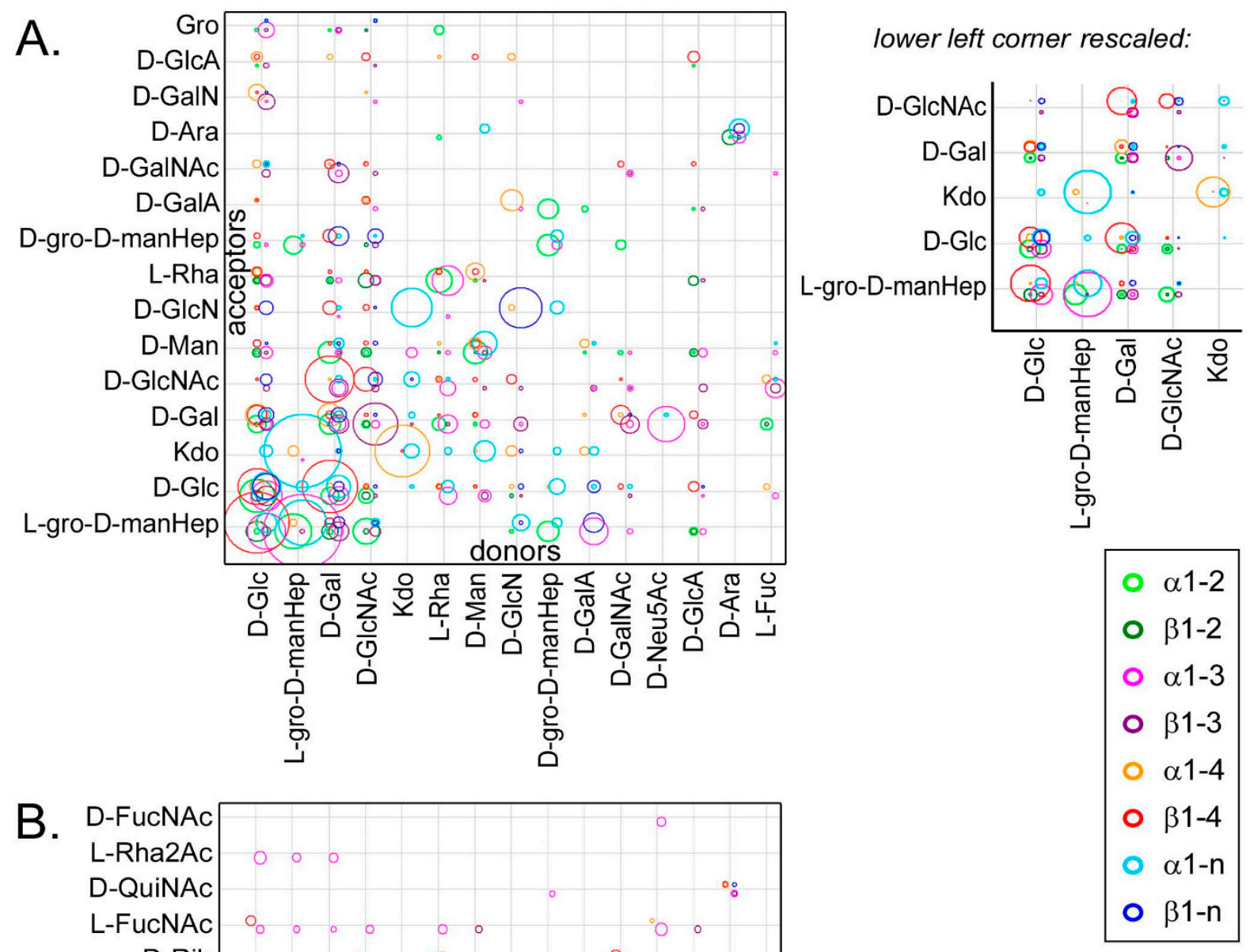

lower left corner rescaled:
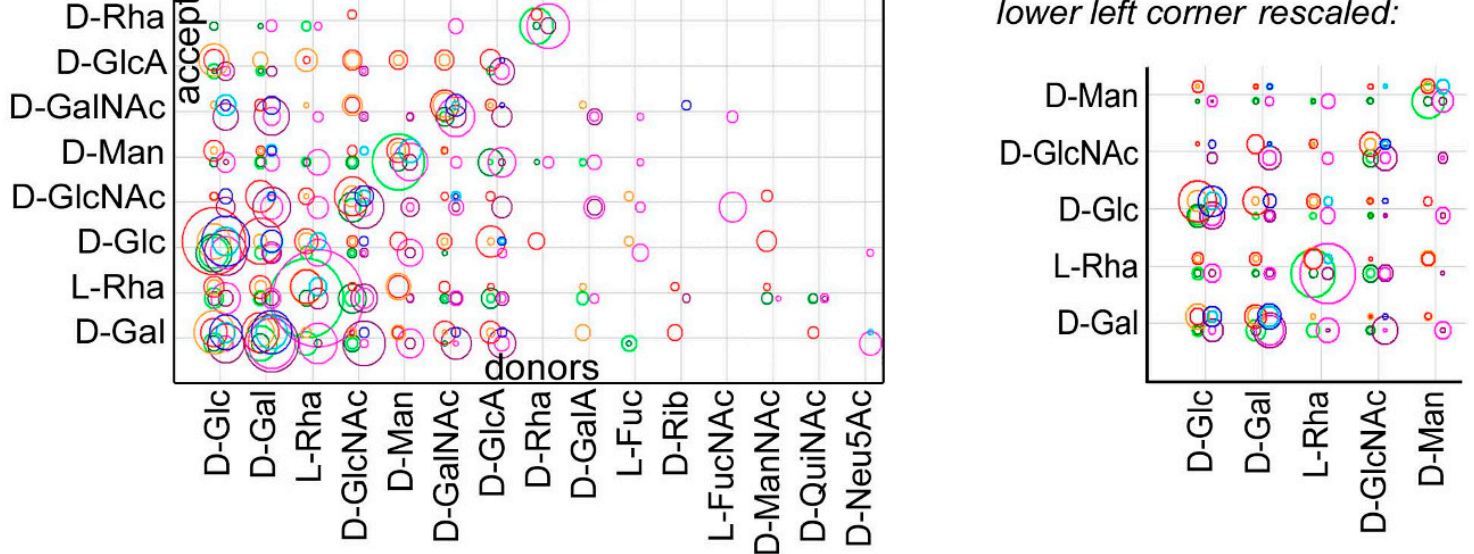

Figure 12

Glycosidic linkages in bacterial carbohydrates. Frequency distribution of specific disaccharide linkages in bacterial carbohydrates. Plotted circle areas represent the relative frequencies for disaccharides formed from the 15 most common donors (children) and 15 most common acceptors (parents) in a defined glycosidic linkage (color code in legend) for bacterial oligomers $(\mathbf{A})$ or polymers $(\mathbf{B})$. The areas of the circles are proportional to the relative abundances of specific disaccharide pairs, normalized to the total number of disaccharide pairs. The linkage codes and plotting offsets are used as in Fig. 12 . The lower left corner of each diagram is plotted at the right with rescaling for better visualization. 
1. The term sequence will be used here to refer to a specific carbohydrate molecule or a glycan obtained from a larger molecule (glycoconjugate). Sequences may be linear or branched. Each database record may refer to either an individual carbohydrate sequence or to a particular glycoconjugate containing a given glycan. Thus, each unique glycan may have multiple database records, one for each different glycoconjugate.

2. A residue is a specific building block, e.g. a monosaccharide, within a carbohydrate sequence, analogous to the amino acid residues in proteins.

3. The term unit will be used to specify the smallest sequence fragment which describes a given carbohydrate molecule or glycan. For a nonrepeating oligomer sequence the unit will be the entire sequence; for polymers built up from repeating subsequences, the unit will be one such subsequence.

4. A branching point is a particular residue to which two or more carbohydrate residues are attached via nonreducing hydroxy functions or other functional groups.

5. A monosaccharide is a unique carbohydrate residue according to the IUPAC definition given above and is specified by the number of carbons, the ring type, the anomeric $(\alpha, \beta)$ and absolute $(D, L)$ configurations, and all primary and secondary modifications. For example, $\alpha$-DGlc $p N, \beta$-D-Glc $p N$ and $\alpha$-D-Glc $p$ NAc are three different monosaccharides derived from glucose.

6. Primary modifications of monosaccharides are those which alter the stereochemical designation or electronic hybridisation state of at least one carbon atom (e.g. deoxy, carboxy, keto, double-bond modifications). Secondary modifications are all modifications which are not primary (substituents such as amino, O-methyl, $O$-acetyl, sulfate, phosphate, etc.).

7. We define the basetype of a monosaccharide to include only those characteristics which specify the order and stereochemical designations of its carbon atom skeleton, i.e., the anomeric and absolute configurations, ring type, and primary modifications. Basetype is not altered by secondary modifications. Thus, $\alpha$-D-Glc $p, \alpha$-D-Glc $p N$ and $\alpha$-DGlcp6S all have the same basetype ( $\alpha$-D-Glc $p$ ) while $\beta$-DGlc $p$ and $\alpha$-D-GlcpA are different basetypes.

8. With respect to common historical usage, the term basic entity will be used to specify the following characteristics of a monosaccharide: the stereochemical configuration (D, L), all primary modifications, and only those secondary modifications involving amine groups, including substituted amines, at any position other than the anomeric carbon. The basic entity definition does not include ano- meric configuration, ring type, or any secondary modifications. Thus, $\alpha-\mathrm{D}-\mathrm{Gal} f \mathrm{~N}$ and $\beta-\mathrm{D}-\mathrm{Gal} p \mathrm{NAc}$ have the same basic entity (D-GalN) while $\beta$-D-Gal $p, \beta$-D-GalpA and $\beta$-DFucp are all different basic entities.

\section{Authors' contributions}

SH carried out the data generation, programmed the analytical procedures and drafted the manuscript together with PVT, who made the statistical analyses and the figures. RR participated in the data generation and made significant contributions to the programming framework. $\mathrm{C}-\mathrm{WvdL}, \mathrm{WEH}$ and YAK participated in the design of the study and helped to draft the manuscript. All authors read and approved the final manuscript.

\section{Additional material}

\section{Additional file 1}

Supplementary Table 1: Abundance of carbohydrate sequences for various taxonomical classes, orders and families.

Click here for file

[http://www.biomedcentral.com/content/supplementary/14726807-8-35-S1.xls]

\section{Additional file 2}

Supplementary Table 2: Abundance of basetypes for bacterial and mammalian carbohydrates.

Click here for file

[http://www.biomedcentral.com/content/supplementary/14726807-8-35-S2.xls]

\section{Additional file 3}

Supplementary Table 3: Abundances of monosaccharide residues found in bacterial and mammalian carbohydrates.

Click here for file

[http://www.biomedcentral.com/content/supplementary/14726807-8-35-S3.xls]

\section{Additional file 4}

Supplementary Table 4: Abundances of all monosaccharide pairs found in bacterial and mammalian carbohydrates.

Click here for file

[http://www.biomedcentral.com/content/supplementary/14726807-8-35-S4.xls]

\section{Additional file 5}

Supplementary Table 5: Comprehensive list of mammalian disaccharide fragments and their relative abundances.

Click here for file

[http://www.biomedcentral.com/content/supplementary/14726807-8-35-S5.rtf]

\section{Additional file 6}

Supplementary Table 6: Comparison of mammalian and bacterial disaccharide fragments and their relative abundances.

Click here for file

[http://www.biomedcentral.com/content/supplementary/14726807-8-35-S6.rtf] 


\section{Acknowledgements}

PT thanks the DKFZ for stipends supporting his stay in Heidelberg. The development of GLYCOSCIENCES.de at the DKFZ was supported by a Research Grant from the Deutsche Forschungsgemeinschaft (DFG BIB 46 HDdkz OI-OI) within the digital library program (SH). The development of the BCSDB (PT) was supported by the International Science and Technology Center (Project I 197p), the Russian Foundation for Basic Research (Project 05-07-90099) and the Russian President Grant Committee (Project MK-2005.1700.4). The EUROCarbDB project, supported by the EU (6th Research Framework Program, RIDS contract number 0I 1952), has also contributed resources to this analysis (RR).

\section{References}

I. Varki A, Cummings R, Esko J, Freeze H, Hart G, Marth J: Essentials of Glycobiology New York: Cold Spring Harbor Laboratory Press; 1999.

2. Ohtsubo K, Marth J: Glycosylation in cellular mechanisms of health and disease. Cell 2006, I 26:855-867.

3. Vollmer W, Blanot D, de Pedro MA: Peptidoglycan structure and architecture. FEMS Microbiology Reviews 2008.

4. Raetz CR, Reynolds CM, Trent MS, Bishop RE: Lipid A modification systems in gram-negative bacteria. Annual Review of Biochemistry 2007, 76:295-329.

5. Raetz CR, Whitfield C: Lipopolysaccharide endotoxins. Annual Review of Biochemistry 2002, 7 I:635-700.

6. Boulnois GJ, Jann K: Bacterial polysaccharide capsule synthesis, export and evolution of structural diversity. Molecular Microbiology 1989, 3:1819-1823.

7. Whitfield C: Biosynthesis and assembly of capsular polysaccharides in Escherichia coli. Annual Review of Biochemistry 2006 75:39-68.

8. Vliegenthart JF: Carbohydrate based vaccines. FEBS Letters 2006, 580:2945-2950.

9. Gagneux P, Varki A: Evolutionary considerations in relating oligosaccharide diversity to biological function. Glycobiology 1999, 9:747-755

10. Stenutz R, Weintraub A, Widmalm G: The structures of Escherichia coli O-polysaccharide antigens. FEMS Microbiology Reviews 2006, 30:382-403

II. Seeberger PH, Werz DB: Synthesis and medical applications of oligosaccharides. Nature 2007, 446: I046-I05I.

12. Toukach F, Knirel Y: New database of bacterial carbohydrate structures. XVIII International Symposium on Glycoconjugates; Florence, Italy 2005:2 16-217 [http://www.glyco.ac.ru/bcsdb/].

13. Luetteke T, Bohne-Lang A, Loss A, Goetz T, Frank M, von der Lieth CW: GLYCOSCIENCES.de: an Internet portal to support glycomics and glycobiology research. Glycobiology 2006, 1 6:7|R-8IR.

14. Doubet S, Bock K, Smith D, Darvill A, Albersheim P: The Complex Carbohydrate Structure Database. Trends in Biochemical Sciences 1989, 14:475-477.

15. Wheeler DL, Chappey C, Lash AE, Leipe DD, Madden TL, Schuler GD, Tatusova TA, Rapp BA: Database resources of the National Center for Biotechnology Information. Nucleic Acids Research 2000, 28:10-14.

16. Herget S, Ranzinger R, Maass K, Lieth C-Wvd: GlycoCT - a unifying sequence format for carbohydrates. Carbohydrate Research 2008, 343:2 162-2171.

17. Hedlund $M$, Tangvoranuntakul P, Takematsu H, Long JM, Housley GD, Kozutsumi Y, Suzuki A, Wynshaw-Boris A, Ryan AF, Gallo RL, Varki $\mathrm{N}$, Varki $\mathrm{A}$ : $\mathbf{N}$-glycolylneuraminic acid deficiency in mice: implications for human biology and evolution. Molecular and Cellular biology 2007, 27:4340-4346.

18. Hoare A, Bittner M, Carter J, Alvarez S, Zaldivar M, Bravo D, Valvano MA, Contreras I: The outer core lipopolysaccharide of Salmonella enterica serovar Typhi is required for bacterial entry into epithelial cells. Infection and Immunity 2006, 74: I555-I 564.

19. He XM, Liu HW: Formation of unusual sugars: mechanistic studies and biosynthetic applications. Annual Review of Biochemistry 2002, 7 I:70I-754.

20. Fusco PC, Farley EK, Huang $\mathrm{CH}$, Moore S, Michon F: Protective meningococcal capsular polysaccharide epitopes and the role of $\mathbf{O}$ acetylation. Clinical Vaccine Immunol 2007, I 4:577-584.
21. Kawagishi S, Araki Y, Ito E: Bacillus cereus autolytic endoglucosaminidase active on cell wall peptidoglycan with $\mathbf{N}$ unsubstituted glucosamine residues. Journal of Bacteriology 1980 , | 4 | : | 37- | 43.

22. Kawano S, Hashimoto K, Miyama T, Goto S, Kanehisa M: Prediction of glycan structures from gene expression data based on glycosyltransferase reactions. Bioinformatics (Oxford, England) 2005 , 2 I:3976-3982.

23. Aoki K, Mamitsuka H, Akutsu T, Kanehisa M: A score matrix to reveal the hidden links in glycans. Bioinformatics 2005, 2 I (8): | 457- 1463.

24. Aoki-Kinoshita K, Ueda N, Mamitsuka H, Kanehisa M: ProfilePSTMM: capturing tree-structure motifs in carbohydrate sugar chains. Bioinformatics (Oxford, England) 2006, I 4:e25-e34.

25. Strecker G, Herlant-Peers MC, Fournet B, Montreul J: Structure of seven oligosaccharides excreted in the urine of a patient with Sandhoff's disease (GM2 gangliosidosis-variant 0 ). European Journal of Biochemistry/FEBS 1977, 8 I: I65-I7I.

26. Jones C: Vaccines based on the cell surface carbohydrates of pathogenic bacteria. Anais da Academia Brasileira de Ciencias 2005, 77:293-324

27. GlycomeDB [http://www.glycome-db.org]

Publish with Biomed Central and every scientist can read your work free of charge

"BioMed Central will be the most significant development for disseminating the results of biomedical research in our lifetime. "

Sir Paul Nurse, Cancer Research UK

Your research papers will be:

- available free of charge to the entire biomedical community

- peer reviewed and published immediately upon acceptance

- cited in PubMed and archived on PubMed Central

- yours - you keep the copyright 